\title{
GALAXY POPULATIONS AND EVOLUTION IN CLUSTERS. IV. DEEP H I OBSERVATIONS OF DWARF ELLIPTICAL GALAXIES IN THE VIRGO CLUSTER
}

\author{
Christopher J. Conselice, ${ }^{1}$ Karen O’Neil, ${ }^{2}$ John S. Gallagher, ${ }^{3}$ and Rosemary F. G. Wyse ${ }^{4}$ \\ Received 2003 January 16; accepted 2003 March 6
}

\begin{abstract}
In this paper we present deep Arecibo H I and WIYN optical observations of Virgo Cluster dwarf elliptical galaxies. Based on this data we argue that a significant fraction of low-mass galaxies in the Virgo Cluster recently underwent evolution. Our new observations consist of $\mathrm{H}$ i $21 \mathrm{~cm}$ line observations for 22 classified $\mathrm{dE}$ galaxies with optical radial velocities consistent with membership in the Virgo Cluster. Cluster members VCC 390 and VCC 1713 are detected with $\mathrm{H}_{\mathrm{I}}$ masses $M_{\mathrm{HI}}=6 \times 10^{7}$ and $8 \times 10^{7} M_{\odot}$, respectively, while $M_{\mathrm{H}_{\mathrm{I}}}$ values in the remaining $20 \mathrm{dE}$ galaxies have upper limits as low as $\sim 5 \times 10^{5} M_{\odot}$. We combine our results with those for 26 other Virgo Cluster dE galaxies with $\mathrm{H}$ I observations in the literature, seven of which have $\mathrm{H}$ I detection claims. New optical images from the WIYN telescope of five of these $\mathrm{H}$ I-detected dE galaxies, along with archival data, suggest that seven of the claimed detections are true $\mathrm{H}$ I detections, yielding $\mathrm{a} \approx 15 \%$ detection rate. These $\mathrm{H}$ I-detected, classified $\mathrm{dE}$ galaxies are preferentially located near the periphery of the Virgo Cluster. Three Virgo dE galaxies have observed H I velocity widths greater than $200 \mathrm{~km} \mathrm{~s}^{-1}$, possibly indicating the presence of a large dark matter content or transient extended $\mathrm{H}$ I. We discuss the possible origins of these objects and argue that they originate from field galaxies accreted onto high angular momentum orbits by Virgo in the last few Gyr. As a result of this, we argue, these galaxies are slowly transformed within the cluster by gradual gas-stripping processes, associated truncation of star formation, and passive fading of stellar populations. Low-mass, early-type cluster galaxies are therefore currently being produced as the product of cluster environmental effects. We utilize our results in a simple model to estimate the recent (past 1-3 Gyr) average mass accretion rate into the Virgo Cluster, deriving a value of $\dot{M} \sim 50 M_{\odot} \mathrm{yr}^{-1}$.
\end{abstract}

Subject headings: galaxies: clusters: individual (Virgo) — galaxies: elliptical and lenticular, cD galaxies: evolution — galaxies: ISM

\section{INTRODUCTION}

Low-mass cluster galaxies (LMCGs), including objects classified as dwarf elliptical galaxies (dE galaxies; Ferguson \& Binggeli 1994), dwarf spheroidal galaxies (Gallagher \& Wyse 1994), and irregular galaxies (Gallagher \& Hunter 1984), hold many clues for understanding galaxy formation and evolution. These are the most common galaxies in the nearby universe (see, e.g., Ferguson \& Binggeli 1994) and may be the first galaxies formed (see, e.g., Blumenthal et al. 1984; White \& Frenk 1991). The relative number densities of dwarf galaxies also increase with local galaxy density (see, e.g., Trentham, Tully, \& Verheijen 2001), with a particularly high abundance found in galaxy clusters, including Virgo (Binggeli, Tammann, \& Sandage 1987, hereafter the Virgo Cluster Catalog, or VCC). As such, LMCGs offer an outstanding opportunity to understand the origin of the lowest mass systems in the universe.

The origins of LMCGs are still a mystery. Star-forming LMCGs, usually classified as dwarf irregular (dIrr) galaxies, are probably recent additions to galaxy clusters (see, e.g., Gallagher \& Hunter 1989; Conselice, Gallagher, \& Wyse 2001a, hereafter Paper I), as indicated by their high gas content, recent star formation, and kinematic characteristics

\footnotetext{
${ }^{1}$ California Institute of Technology, Mail Code 105-24, Pasadena, CA 91125; National Science Foundation Astronomy and Astrophysics Postdoctoral Fellow.

${ }^{2}$ Arecibo Observatory, HC-3, Box 53995, Arecibo, PR 00612.

${ }^{3}$ Department of Astronomy, University of Wisconsin at Madison, 475 North Charter Street, Madison, WI 53706.

${ }^{4}$ Department of Physics and Astronomy, Johns Hopkins University, Bloomberg Center, Baltimore, MD 21218.
}

(Paper I). Quiescent LMCGs, including dE galaxies, ${ }^{5}$ are low-mass $\left(<10^{9} M_{\odot}\right)$, low-luminosity $\left(M_{B}>-17\right)$, low surface brightness $\left(\mu_{B}>24 \mathrm{mag} \operatorname{arcsec}^{-2}\right)$ objects with little to no H I gas (Ferguson \& Binggeli 1994). Despite their faintness, these early-type LMCGs are more common than their star-forming dIrr counterparts and likely contain a mix of stellar population ages and metallicities and thus possibly reflect a variety of origins (see, e.g., Ferguson \& Binggeli 1994; Conselice, Gallagher, \& Wyse 2002, 2003, hereafter Papers II and III, respectively). This paper focuses on understanding the dwarf elliptical-like (dE-like) LMCGs seen in the Virgo Cluster by searching for H I gas that might be left over from their progenitors (see Paper III for a detailed discussion of possible formation scenarios).

Because of their low masses and apparently old stellar populations, dwarf elliptical/spheroidal galaxies in the local universe are outstanding candidates for being among the first galaxies to form. Another scenario is that dE galaxies formed after the cluster's initial collapse (see Papers I and III), possibly originating from accreted field galaxies. It is, however, not yet known with certainty (cf. Vigroux et al. 1986; Gallagher \& Hunter 1989) if there are any galaxies in nearby clusters that are currently undergoing morphological transitions, spiral $\rightarrow \mathrm{dE}, \mathrm{d}$ Irr $\rightarrow \mathrm{dE}$, or spiral + spiral $\rightarrow$

\footnotetext{
${ }^{5}$ The term dwarf elliptical is sometimes used in this paper to describe low-mass objects without recent star formation, and with apparently symmetric structures. Dwarf spheroidal galaxies are a subset of dwarf elliptical galaxies with faint magnitudes and low surface brightnesses (Gallagher \& Wyse 1994). In our view these two terms are interchangeable, and we include both in the general $\mathrm{dE}$ designation. To not bias the interpretation of what Virgo objects with $\mathrm{H}$ I detections are, we use the term low-mass cluster galaxy to describe them, even though these objects have been classified by others (e.g., VCC) as dwarf elliptical galaxies.
} 
elliptical, although inducing these transformations by merging within clusters today is unlikely because of the high relative velocities of cluster members (cf. Conselice, Gallagher, \& Wyse 2001b). Dwarf elliptical formation is, however, potentially still ongoing.

One way to search for objects undergoing formation/evolution into early-type LMCGs is to look for galaxies that morphologically appear as dE galaxies but have properties suggesting recent evolution from star-forming, and possibly more massive, systems such as irregular galaxies or lowmass spiral galaxies. These signs include younger and/or metal-enriched stellar populations (Paper III) and significant atomic gas. We call these galaxies, having properties of several galaxy types, LMCG transition objects. In this paper, we present results from a survey for $\mathrm{H}_{\mathrm{I}} 21 \mathrm{~cm}$ line emission from Virgo early-type LMCGs. We discover two candidate $\mathrm{H}$ I-rich dE galaxies from a sample of 22 observed with the Arecibo $305 \mathrm{~m}$ telescope, which we add to the seven candidate Virgo Cluster dE galaxies with $\mathrm{H}_{\mathrm{I}}$ detections published in the literature. These $\mathrm{H}$ I-detected LMCGs have inferred gas fractions that are high, placing them in the realm of dwarf irregular or Local Group transition-type dwarf galaxies, but have morphologies consistent with dwarf elliptical objects (VCC). Based on an analysis of these properties, we conclude that seven of these LMCGs are likely transition objects, morphologically evolving into early-type dwarf galaxies from star-forming systems.

This paper is organized as follows: $\S 2$ discusses our new observations, $\S 3$ presents our basic results after combining our new data with previously published findings, $\S 4$ gives interpretations of our results, $\S 5$ gives an estimation of the current accretion rate into Virgo, and $\S 6$ is a summary. A Virgo Cluster distance of $18 \mathrm{Mpc}$ is assumed throughout this paper, giving a scale of $\sim 5 \mathrm{kpc} \operatorname{arcmin}^{-1}$.

\section{OBSERVATIONS AND SAMPLE}

The new H I observations we present were taken with the Arecibo $305 \mathrm{~m}$ radio telescope in 2001 April and May. We observed 22 Virgo LMCGs classified as dE galaxies by the VCC (see Table 1). This sample of observed objects was chosen from the list of all classified $\mathrm{dE}$ galaxies in Virgo with known radial velocities, compiled and listed in Paper I. To be observed, an object had to be relatively bright, with an apparent magnitude of $B<17\left(M_{B}<\right.$ -14.0 ). From these systems, a random set of 22 objects classified as dE galaxies, or $23 \%$ of the total number with known radial velocities covering the range of Virgo $\mathrm{dE}$ galaxies (Paper I), was chosen for $21 \mathrm{~cm}$ observations (Table 1). The sample of observed objects is also spread over the entire angular extent of the Virgo Cluster, as defined in the VCC.

Our $21 \mathrm{~cm}$ observations were taken using the Arecibo $L$ Narrow Gregorian receiver. The four Arecibo correlator channels were all centered on the $\mathrm{H} \mathrm{I}(21 \mathrm{~cm})$ line, based on the optically determined radial velocities (Paper I). Two different bandwidths were simultaneously observed, with widths of 6.25 and $12.5 \mathrm{MHz}$, at two different circular polarizations. Observations were made with nine-level sampling, giving each board 2048 channels and providing us with an unsmoothed resolution of 0.65 and $1.3 \mathrm{~km} \mathrm{~s}^{-1}$ for the two different bandwidths. Each target was observed using standard position-switching techniques, with each 5 minute onsource observation followed by a 5 minute "blank-sky" observation, which tracked the same azimuth-zenith angle position of the reflector. Each on+off pair was followed by an observation of a standard noise diode for temperature calibration. All data were obtained at night to eliminate solar interference.

TABLE 1

AREcibo SAMPle

\begin{tabular}{|c|c|c|c|c|c|c|c|}
\hline Name & R.A. (J2000.0) & Decl. (J2000.0) & Morphology ${ }^{a}$ & $m(B)^{\mathrm{a}}$ & $\begin{array}{l}\text { Velocity }^{\mathrm{b}} \\
\left(\mathrm{km} \mathrm{s}^{-1}\right)\end{array}$ & $\begin{array}{c}\text { Integration Time } \\
(\mathrm{ks})\end{array}$ & $\begin{array}{c}3 \sigma \text { Limit }^{\mathrm{c}} \\
\left(\mathrm{mJy} \mathrm{channel}^{-1}\right)\end{array}$ \\
\hline VCC $9 \ldots \ldots \ldots$ & 120922.3 & 135933 & $\mathrm{dE} 1, \mathrm{~N}$ & 13.9 & $1804 \pm 49$ & 2.40 & 0.020 \\
\hline VCC $200 \ldots \ldots$. & 121633.9 & 130156 & $\mathrm{dE} 2, \mathrm{~N}$ & 14.7 & $65 \pm 43$ & 1.80 & 0.044 \\
\hline VCC $303 \ldots \ldots$ & 121842.8 & 054727 & $\mathrm{dE} 2 ?, \mathrm{~N}$ & 15.8 & $2846 \pm 43$ & 2.40 & 0.006 \\
\hline VCC $390 \ldots \ldots$. & 122004.4 & 052457 & dE3 & 16.9 & $2479 \pm 38$ & 1.20 & 0.062 \\
\hline VCC $437 \ldots \ldots$ & 122048.1 & 172916 & $\mathrm{dE} 5, \mathrm{~N}$ & 14.5 & $1474 \pm 46$ & 2.70 & 0.018 \\
\hline VCC $490 \ldots \ldots$. & 122138.8 & 154442 & $\mathrm{dS} 0, \mathrm{~N}$ & 14.3 & $1293 \pm 29$ & 0.60 & 0.056 \\
\hline VCC $543 \ldots \ldots$. & 122219.5 & 144539 & dE5 & 14.8 & $861 \pm 58$ & 5.70 & 0.002 \\
\hline VCC $546 \ldots \ldots$. & 122221.6 & 103607 & dE6 & 15.7 & $2067 \pm 104$ & 2.40 & 0.004 \\
\hline VCC $817 \ldots \ldots$ & 122537.7 & 155006 & $\mathrm{dE} 1$ & 15.0 & $1086 \pm 57$ & 3.00 & 0.016 \\
\hline VCC $856 \ldots \ldots$. & 122557.9 & 100314 & $\mathrm{dE} 1, \mathrm{~N}$ & 14.4 & $972 \pm 32$ & 3.30 & 0.018 \\
\hline VCC $951 \ldots \ldots$. & 122654.4 & 114006 & $\mathrm{dE} 2 \mathrm{p}, \mathrm{N}$ & 14.2 & $2066 \pm 21$ & 1.80 & 0.020 \\
\hline VCC $953 \ldots \ldots$. & 122654.6 & 133357 & $\mathrm{dE} 5 ? \mathrm{p}, \mathrm{N}$ & 15.7 & $-629 \pm 65$ & 2.40 & 0.010 \\
\hline VCC $1261 \ldots$. & 123010.4 & 104646 & $\mathrm{dE} 5, \mathrm{~N}$ & 13.7 & $1850 \pm 30$ & 2.40 & 0.140 \\
\hline VCC $1351 \ldots$. & 123117.5 & 134942 & $\mathrm{dE} 4$ & 16.0 & $187 \pm 48$ & 1.20 & 0.052 \\
\hline VCC $1713 \ldots$. & 123729.0 & 044502 & $\mathrm{dE}$ & 15.1 & $1655 \pm 25$ & 1.50 & 0.068 \\
\hline VCC $1743 \ldots$. & 123806.6 & 100501 & dE6 & 15.1 & $1279 \pm 51$ & 2.40 & 0.008 \\
\hline VCC $1870 \ldots .$. & 124115.3 & 111755 & dE6 & 15.8 & $1617 \pm 27$ & 2.04 & 0.010 \\
\hline VCC $1890 \ldots .$. & 124146.3 & 112915 & $\mathrm{dE} 4 \mathrm{p}$ & 14.8 & $1227 \pm 57$ & 2.04 & 0.026 \\
\hline VCC $1919 \ldots . .$. & 124219.0 & 103404 & $\mathrm{dE} 0, \mathrm{~N}$ & 17.0 & $1869 \pm 54$ & 1.80 & 0.070 \\
\hline VCC $1948 \ldots .$. & 124258.0 & 104055 & dE3 & 15.1 & $1581 \pm 22$ & 2.40 & 0.024 \\
\hline VCC $1971 \ldots .$. & 124331.0 & 110250 & dE3 & 16.6 & $1376 \pm 34$ & 1.20 & 0.052 \\
\hline VCC $2019 \ldots .$. & 124520.4 & 134133 & $\mathrm{dE} 4, \mathrm{~N}$ & 14.6 & $1895 \pm 44$ & 2.70 & 0.022 \\
\hline
\end{tabular}

NoTE.-Units of right ascension are hours, minutes, and seconds, and units of declination are degrees, arcminutes, and arcseconds.

a From the VCC.

${ }^{b}$ From Paper I.

c The $3 \sigma$ detection limit at the $1.3 \mathrm{~km} \mathrm{~s}^{-1}$ resolution. 
During each night, we also observed strong continuum sources to check the observatory-supplied gain curve. All calibration sources were chosen to be nonvariable and to have sizes less than $10 \%$ of the beam width. The flux scales for each source are based on the calibrations from Baars et al. (1977) and Kuehr et al. (1981). We also observed galaxies of small angular size with published $\mathrm{H}$ I line profiles from the list in Lewis, Helou, \& Salpeter (1985). These measurements were done to confirm the accuracy of the internal line flux and frequency calibration. Table 1 lists the integration times and $3 \sigma$ detection limits in the $\mathrm{H}$ I spectrum of each object we observed. On-source integration times ranged from 0.6 to $5.7 \mathrm{ks}$.

We reduced all of our $\mathrm{H}$ i observations using ANALYZ, the Arecibo data analysis program (K. O'Neil 2003, in preparation). To obtain fluxes, velocity widths, and other information for our two new detections, VCC 390 and VCC 1713 (Table 2), first-order baselines were subtracted from the

TABLE 2

Dwarf Elliptical-like Low-Mass Cluster Galaxies in Virgo with H i Observations

\begin{tabular}{|c|c|c|c|c|c|c|c|c|}
\hline Name & Alternate ID & R.A. (J2000.0) & Decl. (J2000.0) & Type $^{\mathrm{a}}$ & $B_{m}$ & $\begin{array}{c}v \\
\left(\mathrm{~km} \mathrm{~s}^{-1}\right)\end{array}$ & Н г Detection & Source \\
\hline VCC $9 \ldots \ldots \ldots$. & IC 3019 & 120922.3 & 135933 & $\mathrm{dE} 1, \mathrm{~N}$ & 13.93 & $1804 \pm 49$ & $\mathrm{~N}$ & 1 \\
\hline VCC $31 \ldots \ldots .$. & & 121056.7 & 091307 & $\mathrm{dE}$ & 14.87 & $2215 \pm 40$ & $\mathrm{Y}$ & 2,3 \\
\hline VCC $168 \ldots \ldots$. & & 121554.3 & 140126 & $\mathrm{dE} 2$ & 17.0 & $682 \pm 10$ & $\mathrm{Y}$ & 4 \\
\hline VCC $200 \ldots \ldots$ & & 121633.9 & 130156 & $\mathrm{dE} 2, \mathrm{~N}$ & 14.69 & $65 \pm 43$ & $\mathrm{~N}$ & 1 \\
\hline VCC $216 \ldots \ldots$. & IC 3097 & 121701.2 & 092432 & $\mathrm{dE} 5, \mathrm{p}, \mathrm{N}$ & 14.9 & $1325 \pm 79$ & $\mathrm{~N}$ & 5,6 \\
\hline VCC $303 \ldots \ldots$. & & 121842.8 & 054727 & $\mathrm{dE} 2 ?, \mathrm{~N}$ & 15.8 & $2846 \pm 43$ & $\mathrm{~N}$ & 1 \\
\hline VCC $390 \ldots \ldots$. & & 122004.4 & 052457 & $\mathrm{dE} 3$ & 16.9 & $2479 \pm 38$ & $\mathrm{Y}$ & 1 \\
\hline VCC $405 \ldots \ldots$. & & 122018.1 & 055957 & $\mathrm{dE} 0$ & 20.0 & 2097 & $\mathrm{Y}$ & 7 \\
\hline VCC $437 \ldots \ldots$. & UGC 7399A & 122048.1 & 172916 & $\mathrm{dE} 5, \mathrm{~N}$ & 14.54 & $1474 \pm 46$ & $\mathrm{~N}$ & 1 \\
\hline VCC $490 \ldots \ldots$. & & 122138.8 & 154442 & $\mathrm{dS} 0, \mathrm{~N}$ & 14.3 & $1293 \pm 29$ & $\mathrm{~N}$ & 1 \\
\hline VCC $543 \ldots \ldots$ & UGC 7436 & 122219.5 & 144539 & $\mathrm{dE} 5$ & 14.77 & $861 \pm 58$ & $\mathrm{~N}$ & 1 \\
\hline VCC $546 \ldots \ldots$. & & 122221.6 & 103607 & dE6 & 15.7 & $2067 \pm 104$ & $\mathrm{~N}$ & 1 \\
\hline VCC $608 \ldots \ldots$. & NGC 4322 & 122301.7 & 155421 & $\mathrm{dE} 4, \mathrm{~N}$ & 14.70 & $1803 \pm 100$ & $\mathrm{Y}$ & 6 \\
\hline VCC $786 \ldots \ldots$ & IC 3305 & 122514.5 & 115057 & $\mathrm{dE} 7, \mathrm{~N}$ & 15.11 & $2388 \pm 30$ & $\mathrm{~N}$ & 8 \\
\hline VCC $797 \ldots \ldots$. & & 122524.2 & 180829 & $\mathrm{dE} 3, \mathrm{~N}$ & 17.0 & $773 \pm 8$ & $\mathrm{Y}$ & 9 \\
\hline VCC $817 \ldots \ldots$ & IC 3313 & 122537.7 & 155006 & $\mathrm{dE} 1$ & 15.0 & $1086 \pm 57$ & $\mathrm{~N}$ & 1 \\
\hline VCC $856 \ldots \ldots$ & IC 3328 & 122557.9 & 100314 & $\mathrm{dE} 1, \mathrm{~N}$ & 14.42 & $972 \pm 32$ & $\mathrm{~N}$ & 1 \\
\hline VCC $917 \ldots \ldots$ & IC 3344 & 122632.4 & 133443 & dE6 & 14.8 & $1375 \pm 66$ & $\mathrm{~N}$ & 6 \\
\hline VCC $940 \ldots \ldots$ & IC 3349 & 122647.1 & 122715 & $\mathrm{dE} 1, \mathrm{~N}$ & 14.78 & $1563 \pm 57$ & $\mathrm{~N}$ & 8 \\
\hline VCC $951 \ldots \ldots$. & IC 3358 & 122654.4 & 114006 & $\mathrm{dE} 2 \mathrm{p}, \mathrm{N}$ & 14.23 & $2066 \pm 21$ & $\mathrm{~N}$ & 1 \\
\hline VCC $953 \ldots \ldots$. & & 122654.6 & 133357 & $\mathrm{dE} 5 ? \mathrm{p}, \mathrm{N}$ & 15.70 & $-629 \pm 65$ & $\mathrm{~N}$ & 1 \\
\hline VCC $965 \ldots \ldots$. & IC 3363 & 122702.9 & 123337 & $\mathrm{dE7}, \mathrm{N}$ & 15.40 & $790 \pm 50$ & $\mathrm{~N}$ & 8 \\
\hline VCC $990 \ldots \ldots$. & IC 3369 & 122717.1 & 160130 & $\mathrm{dE} 4, \mathrm{~N}$ & 14.81 & $1727 \pm 34$ & $\mathrm{~N}$ & 6 \\
\hline VCC $1036 \ldots .$. & NGC 4436 & 122741.6 & 121859 & $\mathrm{dE6}, \mathrm{N}$ & 14.03 & $1163 \pm 50$ & $\mathrm{~N}$ & 6 \\
\hline VCC $1104 \ldots$. & IC 3388 & 122827.9 & 124924 & $\mathrm{dE} 5, \mathrm{~N}$ & 15.31 & $1704 \pm 31$ & $\mathrm{~N}$ & 6 \\
\hline VCC $1122 \ldots$. & IC 3393 & 122841.7 & 125457 & $\mathrm{dE} 7, \mathrm{~N}$ & 14.82 & $436 \pm 29$ & $\mathrm{~N}$ & 6 \\
\hline VCC $1261 \ldots$. & NGC 4482 & 123010.4 & 104646 & $\mathrm{dE} 5, \mathrm{~N}$ & 13.66 & $1850 \pm 30$ & $\mathrm{~N}$ & 1 \\
\hline VCC $1348 \ldots$. & IC 3443 & 123115.7 & 121954 & $\mathrm{dE} 0 \mathrm{p}, \mathrm{N}$ & 15.64 & $1679 \pm 39$ & $\mathrm{~N}$ & 2 \\
\hline VCC $1351 \ldots$. & & 123117.5 & 134942 & $\mathrm{dE} 4$ & 16.0 & $187 \pm 48$ & $\mathrm{~N}$ & 1 \\
\hline VCC $1355 \ldots$. & *IC 3442 & 123120.0 & 140653 & $\mathrm{dE} 2, \mathrm{~N}$ & 14.31 & $1332 \pm 63$ & $\mathrm{~N}$ & 10 \\
\hline VCC $1386 \ldots$. & IC 3457 & 123151.3 & 123921 & $\mathrm{dE} 3, \mathrm{~N}$ & 14.43 & $1426 \pm 60$ & $\mathrm{~N}$ & 6,11 \\
\hline VCC $1491 \ldots$. & *IC 3486 & 123314.0 & 125128 & $\mathrm{dE} 2, \mathrm{~N}$ & 14.8 & $903 \pm 42$ & $\mathrm{~N}$ & 7 \\
\hline VCC $1567 \ldots$. & IC 3518 & 123430.9 & 093728 & $\mathrm{dE} 5, \mathrm{~N}$ & 14.64 & $1440 \pm 55$ & $\mathrm{~N}$ & 6,11 \\
\hline VCC $1713 \ldots$. & & 123729.0 & 044502 & $\mathrm{dE}$ & 15.1 & $1655 \pm 25$ & $\mathrm{Y}$ & 1 \\
\hline VCC $1743 \ldots$. & IC 3602 & 123806.6 & 100501 & $\mathrm{dE} 6$ & 15.1 & $1279 \pm 51$ & $\mathrm{~N}$ & 1 \\
\hline VCC $1826 \ldots .$. & IC 3633 & 124011.2 & 095346 & $\mathrm{dE} 2, \mathrm{~N}$ & 14.87 & $2033 \pm 30$ & $\mathrm{~N}$ & 6 \\
\hline VCC $1857 \ldots .$. & IC 3647 & 124053.2 & 102833 & $\mathrm{dE} 4, \mathrm{~N} ?$ & 14.33 & $634 \pm 69$ & $\mathrm{~N}$ & 6,11 \\
\hline VCC $1870 \ldots$. & & 124115.3 & 111755 & dE6 & 15.8 & $1617 \pm 27$ & $\mathrm{~N}$ & 1 \\
\hline VCC $1876 \ldots$. & IC 3658 & 124120.4 & 144202 & $\mathrm{dE} 5, \mathrm{~N}$ & 14.85 & $45 \pm 49$ & $\mathrm{~N}$ & 6 \\
\hline VCC $1890 \ldots .$. & IC 3665 & 124146.3 & 112915 & $\mathrm{dE} 4 \mathrm{p}$ & 14.84 & $1227 \pm 57$ & $\mathrm{~N}$ & 1 \\
\hline VCC $1910 \ldots$. & IC 809 & 124207.8 & 114516 & $\mathrm{dE} 1, \mathrm{~N}$ & 14.17 & $206 \pm 26$ & $\mathrm{~N}$ & 6 \\
\hline VCC $1919 \ldots$ & & 124219.0 & 103404 & $\mathrm{dE} 0, \mathrm{~N}$ & 17.0 & $1869 \pm 54$ & $\mathrm{~N}$ & 1 \\
\hline VCC $1948 \ldots . .$. & & 124258.0 & 104055 & $\mathrm{dE} 3$ & 15.1 & $1581 \pm 22$ & $\mathrm{~N}$ & 1 \\
\hline VCC $1949 \ldots$ & NGC 4640 & 124258.1 & 121710 & $\mathrm{dE6}, \mathrm{N}$ & 14.19 & $2077 \pm 75$ & $\mathrm{Y}$ & $6,8,12$ \\
\hline VCC $1971 \ldots$. & & 124331.0 & 110250 & $\mathrm{dE} 3$ & 16.6 & $1376 \pm 34$ & $\mathrm{~N}$ & 1 \\
\hline VCC $2019 \ldots$. & IC 3735 & 124520.4 & 134133 & $\mathrm{dE} 4, \mathrm{~N}$ & 14.55 & $1895 \pm 44$ & $\mathrm{~N}$ & 1 \\
\hline VCC $2050 \ldots$. & IC 3779 & 124720.7 & 120959 & $\mathrm{dE} 5, \mathrm{~N}$ & 15.2 & $1367 \pm 75$ & $\mathrm{~N}$ & 6 \\
\hline VCC $2062 \ldots$. & & 124759.9 & 105833 & $\mathrm{dE}$ & 19.0 & $1146 \pm 8$ & $\mathrm{Y}$ & 13,14 \\
\hline
\end{tabular}

NOTE.- Units of right ascension are hours, minutes, and seconds, and units of declination are degrees, arcminutes, and arcseconds.

a Morphological types are from the estimates given by the VCC.

REFERENCES.- (1) This paper; (2) van Driel et al. 2000; (3) van Zee et al. 1995; (4) Hoffman et al. 1987; (5) Bothun et al. 1985; (6) Huchtmeier \& Richter 1986; (7) Duprie \& Schneider 1996; (8) Haynes \& Giovanelli 1986; (9) Burstein et al. 1987; (10) Lake \& Schommer 1984; (11) Schneider et al. 1990; (12) Bottinelli et al. 1990; (13) Cayatte et al. 1990; (14) Hoffman et al. 1993. 

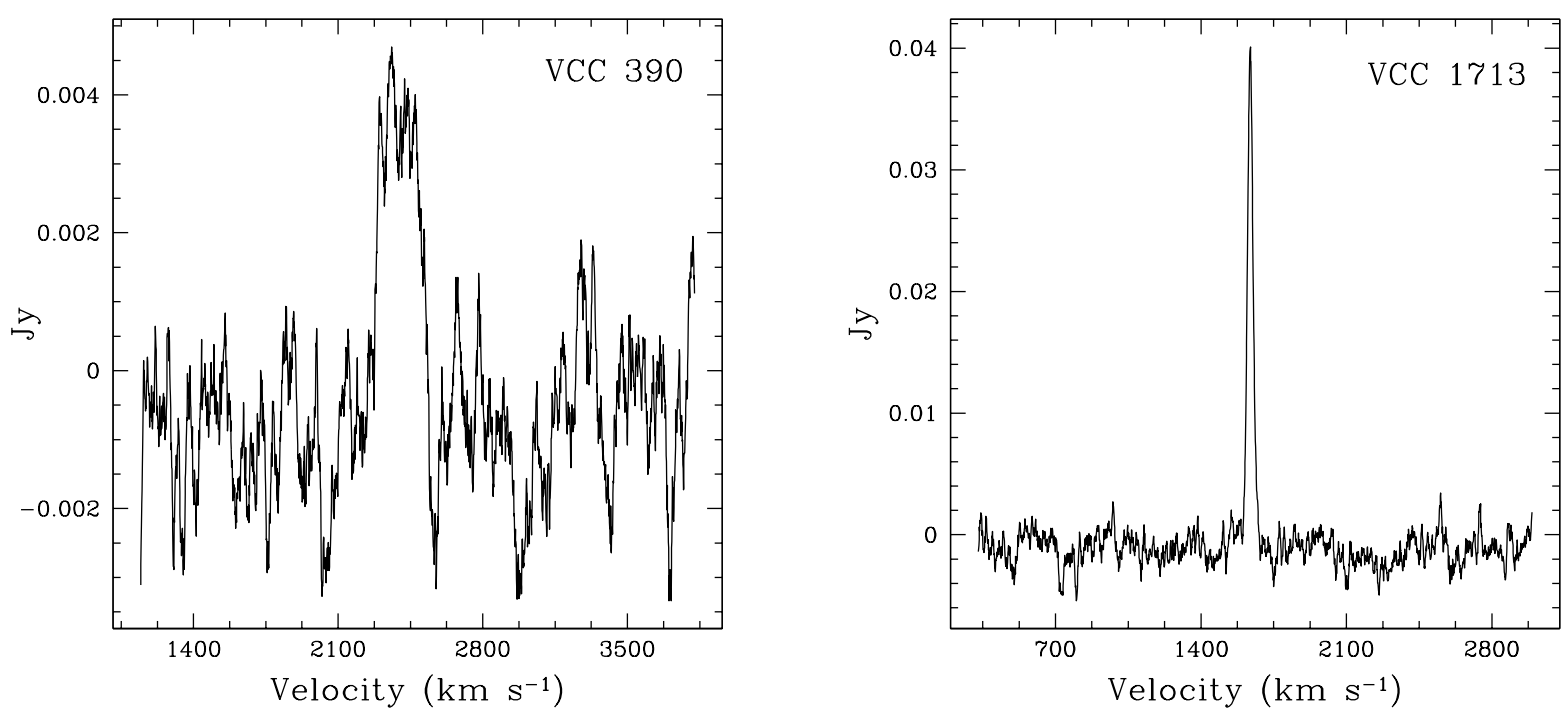

FIG. 1.-Arecibo H i spectra of our two objects with H I detections, VCC 390 and VCC 1713

observed spectra. The baselines were fitted interactively and then removed from the $\mathrm{H}$ I line profile. Both single and double Gaussians were then fitted to the $\mathrm{H}$ I lines. These profiles were fitted within the area that occupies the $\mathrm{H}$ I emission. That is, we start and end the fit where the line distinguishes itself from the noise. Since this is ultimately somewhat of a subjective process, we repeated it several times to obtain an estimated random error. This fitting process gives line widths at $20 \%$ and $50 \%$ of the peak brightness, as well as the total flux in each $\mathrm{H} \mathrm{I}$ line.

\section{RESULTS}

\subsection{H I Properties}

Of the 22 galaxies we observed at Arecibo, only two have significant $\mathrm{H}$ I detections (Fig. 1). This is not an exceptionally low rate, since many previous observations also found few to no detections of $\mathrm{H}$ I in Virgo Cluster galaxies classified as dE (see, e.g., Huchtmeier \& Richter 1986; Bothun et al. 1985). We supplemented our sample by performing a literature search to find other classified Virgo $\mathrm{dE}$ galaxies with $\mathrm{H}$ I detections; a further seven objects with $21 \mathrm{~cm}$ detec- tion claims were retrieved. We discuss these seven objects in $\S 3.5$. Table 2 lists all of the Virgo Cluster dwarf galaxies classified as $\mathrm{dE}$ observed to date at $21 \mathrm{~cm}$.

Since the new observations presented here are the most sensitive ever taken, we might expect a higher fraction of detections if $\mathrm{H}$ I gas was commonly found in early-type LMCGs. There are 97 Virgo galaxies classified as dwarf elliptical with measured stellar radial velocities (Paper I), and $48(49 \%)$ of these have been observed in the $21 \mathrm{~cm}$ line. Of these, nine (19\% of the total) have claimed H I detections. Through the following analysis we conclude that one of these detections is not significant enough to be considered real (VCC 405), and we argue that the other is a dwarf irregular galaxy (VCC 2062). All of the published H I detections, including our two new objects, are listed in Table 3.

\subsubsection{H i Masses}

We used the flux of the $21 \mathrm{~cm}$ line to determine $\mathrm{H}$ I gas masses for each detected LMCG through the formula

$$
M_{\mathrm{H} \text { I }}\left(M_{\odot}\right)=2.36 \times 10^{5} D(\mathrm{Mpc})^{2} \int S(\nu) d \nu
$$

TABLE 3

Properties of H i-Detected LMCGs

\begin{tabular}{|c|c|c|c|c|c|c|c|c|c|c|}
\hline Name & $M_{B}$ & $\begin{array}{c}W_{50} \\
\left(\mathrm{~km} \mathrm{~s}^{-1}\right)\end{array}$ & $\begin{array}{c}W_{20} \\
\left(\mathrm{~km} \mathrm{~s}^{-1}\right)\end{array}$ & $\begin{array}{c}\int S d v \\
\left(\mathrm{Jy} \mathrm{km} \mathrm{s}^{-1}\right)\end{array}$ & $\begin{array}{c}M_{\mathrm{H} \mathrm{I}_{\mathrm{I}}} \\
\left(\times 10^{9} M_{\odot}\right)\end{array}$ & $\begin{array}{l}M_{\mathrm{H} \mathrm{I}} / L_{\mathrm{B}} \\
\left(M_{\odot} / L_{\odot}\right)\end{array}$ & $\begin{array}{c}R^{\mathrm{a}} \\
(\mathrm{kpc})\end{array}$ & $\begin{array}{c}M_{\mathrm{dyn}} \\
\left(\times 10^{9} M_{\odot}\right)\end{array}$ & $f_{\mathrm{gas}}^{\mathrm{b}}$ & $\begin{array}{c}M_{\mathrm{dyn}} / L_{B} \\
M_{\odot} / L_{\odot}\end{array}$ \\
\hline VCC $31 \ldots \ldots \ldots . .$. & -16.4 & 142 & 132 & 1.53 & 0.12 & 0.21 & 1.2 & 1.4 & 0.08 & 2.4 \\
\hline VCC $168 \ldots \ldots \ldots$ & -14.3 & 50 & 64 & 0.34 & 0.03 & 0.33 & 1.4 & 0.2 & 0.12 & 2.5 \\
\hline VCC $390 \ldots \ldots . .$. & -14.4 & $285 \pm 7$ & $286 \pm 5$ & $0.8 \pm 0.01$ & 0.06 & 0.68 & 0.8 & 4 & 0.02 & 45 \\
\hline $\operatorname{VCC} 405^{\mathrm{c}} \ldots \ldots$. & -11.5 & 282 & $\ldots$ & 1.1 & 0.08 & 8.4 & 0.5 & 2.2 & 0.04 & 218 \\
\hline VCC $608 \ldots \ldots . .$. & -16.6 & 88 & 195 & 16 & 1.22 & 1.8 & 3.8 & 1.7 & 0.72 & 2.5 \\
\hline VCC $797 \ldots \ldots . .$. & -14.3 & 28 & $\ldots$ & 0.40 & 0.03 & 0.38 & 1.2 & 0.1 & 0.57 & 0.6 \\
\hline VCC $1713 \ldots \ldots$ & -16.2 & $46 \pm 8$ & $60 \pm 6$ & $1.1 \pm 0.004$ & 0.08 & 0.20 & 1.4 & 0.2 & 0.56 & 0.4 \\
\hline VCC 1949 ....... & -17.1 & 233 & 304 & 5.5 & 0.42 & 0.38 & 6.3 & 79.0 & 0.02 & 18 \\
\hline VCC $2062^{\mathrm{d}} \ldots .$. & -12.3 & 87 & $\ldots$ & 7.2 & 0.55 & 55 & 1.8 & 0.8 & 0.72 & 78 \\
\hline
\end{tabular}

a Radii measurements are from the VCC.

${ }^{\mathrm{b}}$ Fraction of gas in $\mathrm{H}_{\mathrm{I}}$, defined as $M_{\mathrm{H}} / M_{\text {dyn }}$.

${ }^{\mathrm{c}}$ Likely a spurious $\mathrm{H}$ I detection (see $\S 3.5 .3$ ).

$\mathrm{d}$ This object is probably a pure dwarf irregular (§ 3.5 .7$)$. 
where $D$ is the distance to the galaxy in Mpc, in our case assumed fixed at $18 \mathrm{Mpc}$, and $\int S(\nu) d \nu$ is the integral of the flux in units of Jy km s${ }^{-1}$. For the undetected galaxies we observed at Arecibo, we set limits on $\mathrm{H}$ I masses (Table 1) by assuming a $3 \sigma$ detection limit over $60 \mathrm{~km} \mathrm{~s}^{-1}$. This provides an average $\mathrm{H}$ I upper limit mass of $\sim 8 \times 10^{6} M_{\odot}$. Our deepest observation was for the galaxy VCC 543, where we reach a $3 \sigma$ limit in the $12.5 \mathrm{MHz}$ band of $0.002 \mathrm{mJy}_{\text {channel }}{ }^{-1}$, corresponding to a $3 \sigma$ upper mass limit of $4.6 \times 10^{5} M_{\odot}$.

With these limits we are close to the detectability threshold of some Local Group dwarf elliptical and spheroidal galaxies. For example, the dwarf elliptical galaxies NGC 205 and NGC 185 have $\mathrm{H}$ I masses of $3.4 \times 10^{5}$ and $1.3 \times 10^{5} M_{\odot}$, respectively (Young \& Lo 1997; Johnson \& Gottesman 1993). Dwarf irregular galaxies such as IC 10 and IC 1613, with $\mathrm{H}$ I masses of $1.2 \times 10^{8}$ and $6.5 \times 10^{7} M_{\odot}$, respectively (Huchtmeier 1979; Volders \& Högbom 1961), would easily be detected in our survey. Even the faintest Local Group dwarf irregular galaxy, the Sagittarius DIG, has an $\mathrm{H}$ I gas content of $1.1 \times 10^{7} M_{\odot}$ and would be easily detectable at the distance of Virgo in our survey.

However, a few Local Group dwarf spheroidal galaxies, such as NGC 147, still remain undetected in $\mathrm{H} \mathrm{I}$, down to a limit of $\sim 3 \times 10^{3} M_{\odot}$ (Young 1999), and many of the objects we observe with no detections could have equally low $\mathrm{H}$ I gas masses. Fainter dwarf spheroidal galaxies, such as Fornax and Leo II, also have extremely low H I mass upper limits of less than $10^{4} M_{\odot}$ (Young 1999).

\subsection{Optical Images}

Figure 2 shows the second-generation Digital Sky Survey (DSS) images for all nine early-type LMCGs with reported $\mathrm{H}$ I detections. This field size was chosen to match the Arecibo beam, but detections using other telescopes had different beam sizes. In several cases, e.g., VCC 405, nothing obvious is seen, demonstrating that some objects are very faint. In most cases, however, it is clear that these LMCGs are smooth objects with very little structural evidence for active star formation, consistent with their classification as dwarf elliptical galaxies by the VCC.

We also acquired WIYN $3.5 \mathrm{~m}$ broadband Harris $R$ images for five of the nine Virgo H I-detected LMCGs (VCC 31, VCC 168, VCC 390, VCC 797, VCC 1713). These shallow images were taken under nonphotometric conditions using the Mini-Mosaic CCD, which has a field of view 9! $6 \times 9$ ' 6 . Mini-Mosaic consists of two SITe $4096 \times 2048$ pixel CCDs separated by a gap of $5^{\prime \prime}$ and a pixel scale of 0 ". 14 pixel $^{-1}$ (see Paper II for a further description of MiniMosaic). The typical exposure time for these five images was
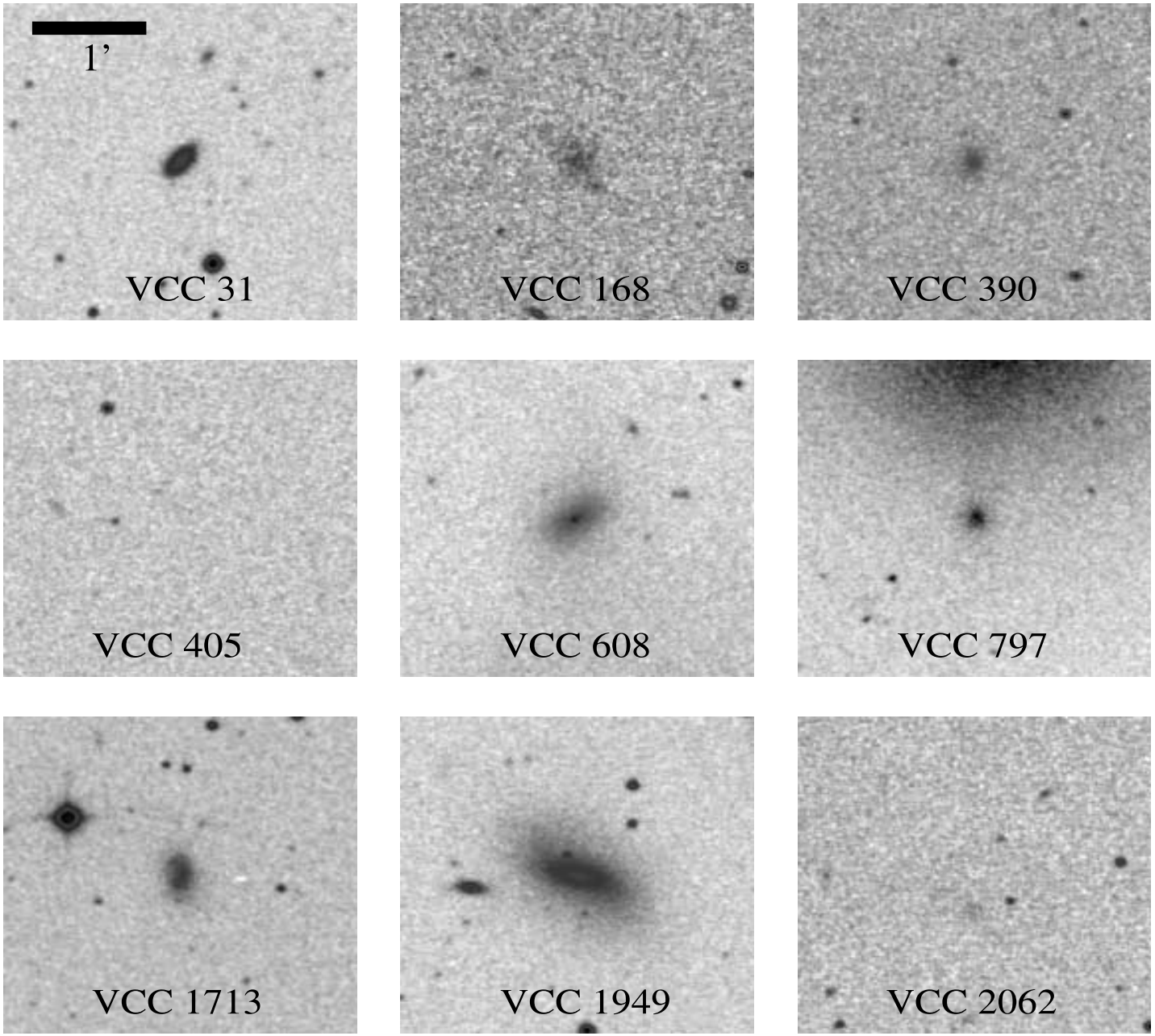

FIg. 2.-Palomar Sky Survey images centered on the nine candidate dE galaxies with H I detections. The size of these images matches the beam of the Arecibo telescope at the $21 \mathrm{~cm}$ wavelength $\left(\sim 3^{\prime}\right)$. 

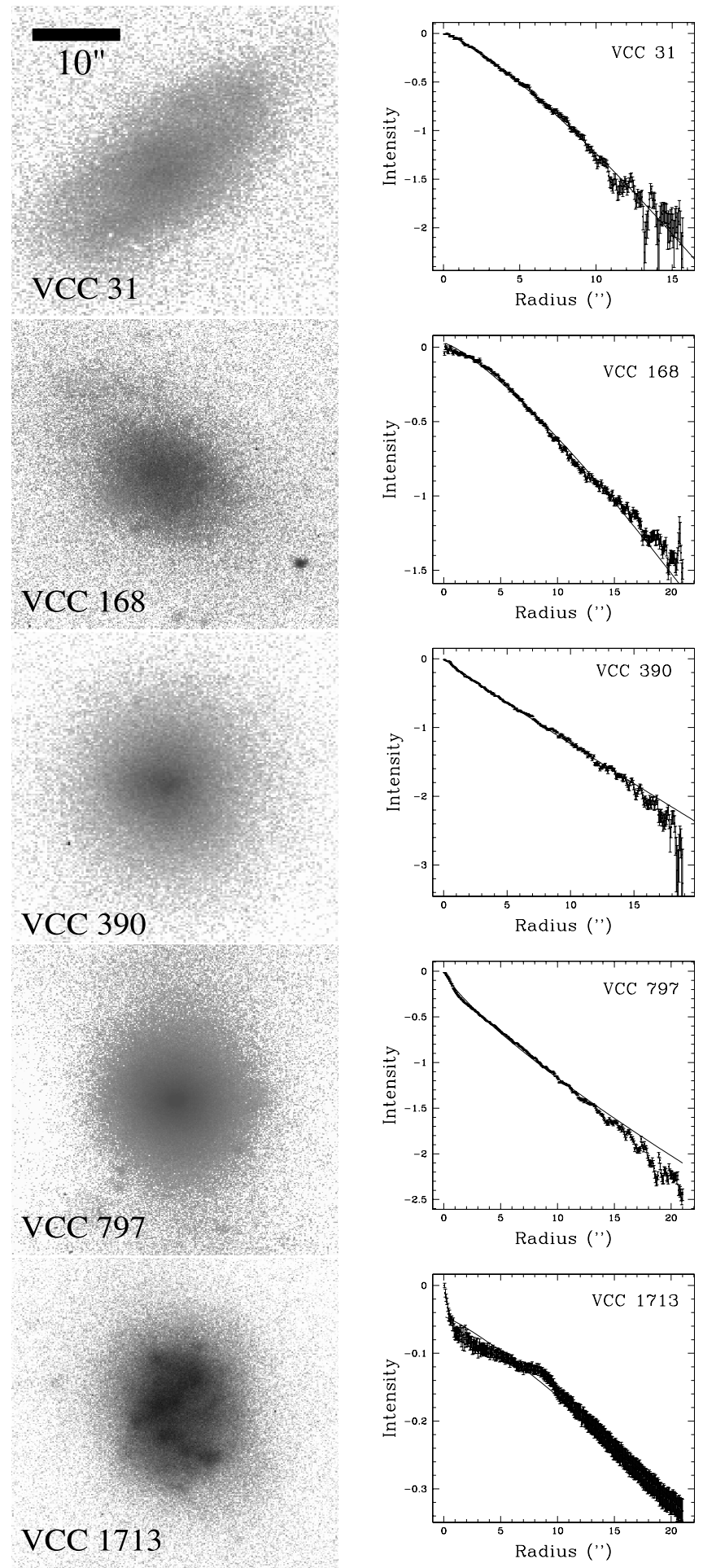

FIG. 3.-Images and surface brightness profiles for the five objects detected in H I, imaged with the WIYN $3.5 \mathrm{~m}$ telescope in the $R$ band. The intensity is lognormalized to zero at the peak intensity, and all images are $\sim 35^{\prime \prime}$ on a side, with the scale indicated by the bar on the top panel.

$500 \mathrm{~s}$, suitable for obtaining morphological and structural information.

The five $\mathrm{H}$ I-detected LMCGs imaged with WIYN are shown in Figure 3. With the possible exception of VCC 1713 , these galaxies all appear to be quiescent, early-type LMCGs, confirming the impression from Figure 2. Figure 3 also shows the surface brightness profiles for these five
LMCGs with an arbitrary normalization. Table 4 lists the quantitative morphological properties for these galaxies, including the asymmetry $A$ (Conselice 1997; Conselice, Bershady, \& Gallagher 2000a; Conselice, Bershady, \& Jangren 2000b), concentration $C$ (Bershady, Jangren, $\&$ Conselice 2000), clumpiness index $S$ (Conselice 2003), as well as the Sérsic profile index and scale radius $n$ and $r_{0}$, respectively, according to the formula $I=I_{0} \exp \left[-\left(r / r_{0}\right)^{1 / n}\right]$ (see Paper III for details).

The asymmetry parameter $A$ is a measure of how asymmetric a galaxy is, found by quantifying the residuals after rotating a galaxy's image $180^{\circ}$ by its center and subtracting this new image from the original (Conselice et al. 2000b). Most early-type galaxies have $A \sim 0$. The concentration parameter $C$ is a measure of how concentrated the light in a galaxy is toward its center. Most elliptical galaxies have $C>3.5$, while disks and dwarf galaxies have lower values in the range $2<C<3$ (Bershady et al. 2000). The clumpiness index $S$ is found by quantifying the fraction of spatial highfrequency light in a galaxy. Most early-type galaxies have very little high spatial frequency structure and thus have clumpiness values $S \sim 0$. Table 4 is consistent with the visual impression of these as early-type galaxies.

\subsection{Dynamical Masses and $M_{d y n} / L_{B}$ Ratios}

Although we cannot make any firm dynamical mass claims, we can compute some estimates based on the likely physical conditions of each LMCG with an $\mathrm{H}$ I detection (Table 3). A major problem in computing dynamical masses for these galaxies is deciding what radius corresponds to the measured velocity width and what component of the singleGaussian line widths are due to rotation, velocity anisotropy, and random turbulent motion. To estimate dynamical masses, we assume that the $\mathrm{H}$ I velocity width represents rotation on orbits that are nearly circular, plus some turbulence. Observations show that the $\mathrm{H}$ I sizes of galaxies can be much bigger than their optical sizes (see, e.g., Broeils 1992; van Zee, Skillman, \& Salzer 1998), although some Virgo galaxies have truncated $\mathrm{H}$ I profiles (see, e.g., Haynes \& Giovanelli 1986). For a size, we use the optical radius estimates for these galaxies given in the VCC and listed in Table 3 . We also assume the circular velocity $\left(V_{\text {rot }}\right)$ to be half the $\mathrm{H}$ I line velocity width at $50 \%$ maximum, $W_{50}$, uncorrected for inclination, from our $\mathrm{H}$ i spectroscopy. Normally we would use the $W_{20}$ velocity widths, but these values are not available for many of the galaxies listed in Table 2. Using $W_{50}$ instead of $W_{20}$ reduces the dynamical mass and massto-light ratios by an average of $\sim 70 \%$ and increases the $\mathrm{H}$ I mass fraction by the same amount (Table 3). We compute lower limits to dynamical masses using the equation

$$
M_{\text {dyn }} \geq V_{\text {rot }}^{2}(R / G) \sim 0.25 W_{50}^{2}(R / G),
$$

where we have used the approximation $V_{\text {rot }}=W_{50} / 2$ with the derived masses listed in Table 3 . The radii we use are from the VCC and are also listed in Table 3. From these values we are able to compute dynamical mass-to-light ratios $\left(M_{\mathrm{dyn}} / L_{B}\right)$ and gas mass fractions $\left(f_{\mathrm{gas}}=M_{\mathrm{H}_{\mathrm{I}}} / M_{\mathrm{dyn}}\right)$, which are listed in Table 3. Several of these galaxies have very high mass-to-light ratios, as discussed below, while others have rather modest values, with $M_{\mathrm{dyn}} / L_{B} \sim 1$ in solar units. The highest $M_{\mathrm{dyn}} / L_{B}$ values are found for VCC 405 and VCC 2062, which we conclude in $\S 3.5$ are likely not dwarf elliptical galaxies or real detections. We also discuss 
TABLE 4

Quantitative Morphological Properties of H i-Detected LMCGs

\begin{tabular}{|c|c|c|c|c|c|c|}
\hline Name $^{\mathrm{a}}$ & $A^{\mathrm{b}}$ & $C^{\mathrm{c}}$ & $S^{\mathrm{d}}$ & $n^{\mathrm{e}}$ & $\begin{array}{c}r_{0}{ }^{\mathrm{e}} \\
(\operatorname{arcsec})\end{array}$ & $\begin{array}{c}r_{0} \mathrm{e} \\
(\mathrm{kpc})\end{array}$ \\
\hline VCC $31 \ldots \ldots .$. & $-0.02 \pm 0.10$ & 2.44 & $0.02 \pm 0.13$ & $1.27 \pm 0.01$ & $4.4 \pm 0.03$ & $0.38 \pm 0.00$ \\
\hline VCC $168 \ldots \ldots$. & $0.00 \pm 0.17$ & 2.63 & $-0.04 \pm 0.09$ & $1.28 \pm 0.02$ & $7.4 \pm 0.09$ & $0.64 \pm 0.01$ \\
\hline VCC $390 \ldots \ldots$ & $-0.08 \pm 0.11$ & 2.95 & $-0.14 \pm 0.11$ & $0.95 \pm 0.01$ & $3.3 \pm 0.04$ & $0.29 \pm 0.00$ \\
\hline VCC $797 \ldots \ldots$ & $-0.02 \pm 0.05$ & 2.69 & $0.16 \pm 0.06$ & $0.80 \pm 0.01$ & $2.8 \pm 0.07$ & $0.24 \pm 0.01$ \\
\hline VCC $1713 \ldots$. & $-0.04 \pm 0.08$ & 2.54 & $0.07 \pm 0.05$ & $1.20 \pm 0.03$ & $29.8 \pm 0.33$ & $2.6 \pm 0.03$ \\
\hline
\end{tabular}

a Object name from the VCC.

b Asymmetry index, A, as defined and measured in Conselice et al. 2000a. See Conselice et al. 2000b and Conselice 2003 for further interpretations of this index.

${ }^{c}$ Concentration index, $C$, as defined in Bershady et al. 2000. Higher values of this index $(\sim 4)$ are typically found for giant elliptical galaxies, while disk and dwarf galaxies have values $\sim 2-3$.

d Clumpiness index, $S$, as defined in Conselice 2003.

e The Sérsic index, $n$, and scale length, $r_{0}$, as defined in $\S 3.2$. Scale lengths are converted from arcseconds into kiloparsecs assuming a distance of $18 \mathrm{Mpc}$ to the Virgo Cluster.

alternative processes that can potentially create large $\mathrm{H}$ I velocity widths, besides deep gravitational potential wells.

\subsection{New Detections}

In this section we discuss the $21 \mathrm{~cm}$ and optical properties for each of our new Arecibo $\mathrm{H}$ I detections and give some possible interpretations of their measurements. We wish to stress that both of these detections have only occurred with Arecibo and its large beam, and confusion with other galaxies could be an issue (Hibbard \& Sansom 2003).

\subsubsection{VCC 390}

In the DSS and WIYN images (Figs. 2 and 3) VCC 390 appears as a small, compact galaxy. Using the WIYN image of this galaxy (Fig. 3), its scale length is fitted as $0.29 \pm 0.003$ $\mathrm{kpc}$ (Table 4). This object is classified as a dE3 in the VCC and has an absolute magnitude of $M_{B}=-14.4$ and low asymmetry, low concentration, and low clumpiness values (Table 4), all consistent with it being a dwarf elliptical galaxy (Conselice 2003). It is also fairly well fitted by an exponential profile with a Sérsic index, $n=0.95 \pm 0.01$. This galaxy is, however, fainter toward its outer parts than its Sérsic fit.

The observed wavelength of the $\mathrm{H}$ I line for VCC 390 gives a heliocentric radial velocity of $2400 \pm 10 \mathrm{~km} \mathrm{~s}^{-1}$ (Fig. 1), in relatively good agreement with its optical radial velocity, $2479 \pm 38 \mathrm{~km} \mathrm{~s}^{-1}$, computed through cross-correlation fits of absorption features in the optical VCC 390 spectrum shown in Figure 4. This is a WIYN Hydra Multiobject Spectrograph spectrum taken in 1999 April as part of a radial velocity survey (Paper I). This is a typical early-type galaxy spectrum showing absorption features such as the Mgb triplet at $5175 \AA$. No emission lines are seen; these would be an indication of active star formation, as seen in Virgo dwarf irregular galaxies (see, e.g., Gallagher \& Hunter 1989; Heller, Almoznino, \& Brosch 1999). The spectrum of VCC 390 is also similar to spectra of other Virgo dwarf elliptical galaxies (see Paper I).

The H I line of VCC 390 presents an interesting puzzle due to its large velocity width (Table 3). The $20 \%$ and $50 \%$ FWHM velocities are $286 \pm 5$ and $285 \pm 7 \mathrm{~km} \mathrm{~s}^{-1}$, respectively, with a total integrated $\mathrm{H}$ I flux of $0.8 \pm 0.01 \mathrm{Jy} \mathrm{km}$ $\mathrm{s}^{-1}$. It is only one of three galaxies in our sample that has a velocity width greater than $200 \mathrm{~km} \mathrm{~s}^{-1}$. Because of its faint magnitude, it also has an inferred high dynamical mass-to-light ratio $\left(M_{\mathrm{dyn}} / L_{B}\right)$ of $\sim 45$ in solar units (Table $3)$. However, it is possible that some of this line emission originates from contamination in the sidelobes due to the nearby galaxy NGC 4277, which is at a velocity of $2504 \mathrm{~km}$ $\mathrm{s}^{-1}$ and has an $\mathrm{H}$ I line profile that overlaps in velocity with VCC 390's (see van Driel et al. 2000). We investigated this possibility by overlaying the Arecibo beam map onto a DSS image of VCC 390 and NGC 4277. We found that the outer lobes of the profile as centered on VCC 390 slightly overlapped with NGC 4277. Short of this, the large velocity width could be the result of gas stripping. It is, however, impossible to determine using our current data how much these sources are contributing to the VCC 390 signal. Understanding this will require mapping the spatial extent

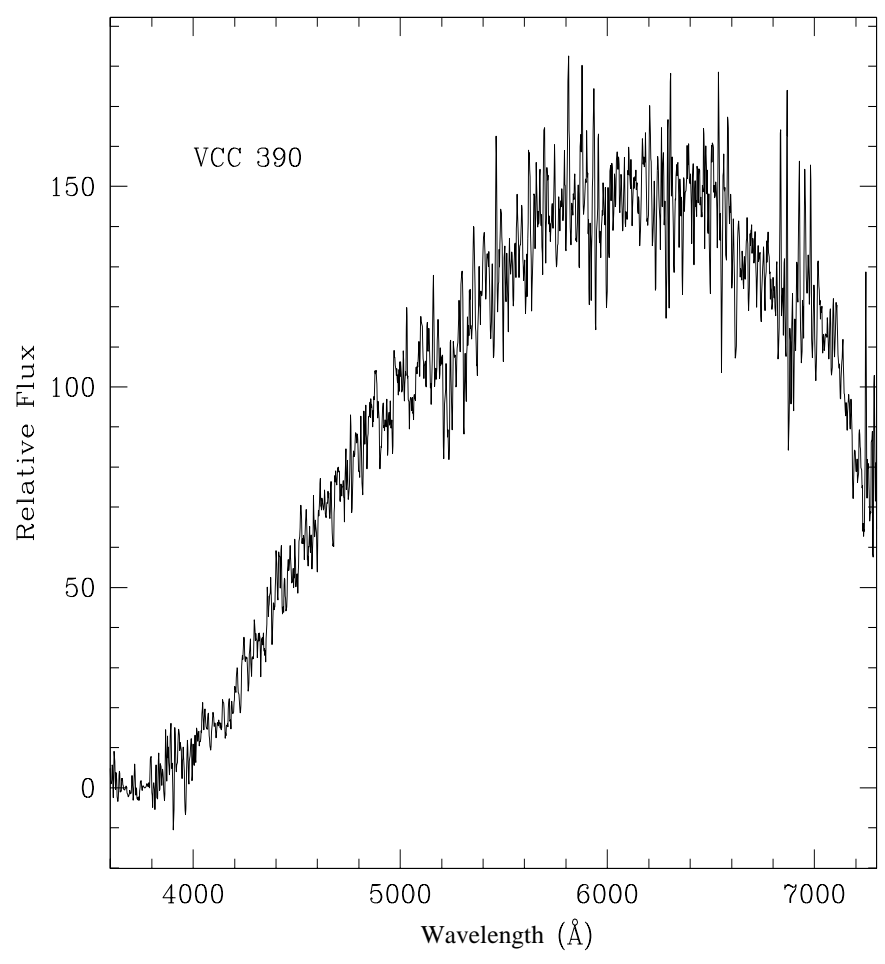

FIG. 4.-Optical spectrum of the dwarf elliptical galaxy VCC 390, taken with the WIYN 3.5 m telescope with an arbitrary flux normalization. 
of the $\mathrm{H}$ I distribution of VCC 390 and comparing it with that of NGC 4277.

\subsubsection{VCC 1713}

The other low-mass galaxy with a new $\mathrm{H}$ I detection is VCC 1713. Compared to VCC 390, VCC 1713 has a narrower velocity $\mathrm{H}$ I profile, with $W_{50}=46 \pm 8 \mathrm{~km} \mathrm{~s}^{-1}$ and $W_{20}=60 \pm 6 \mathrm{~km} \mathrm{~s}^{-1}$, more commonly expected for a lowmass galaxy. The total $\mathrm{H}$ I flux is slightly higher than that of VCC 390, with an integrated value of $1.1 \mathrm{Jy} \mathrm{km} \mathrm{s}^{-1}$, giving a total $\mathrm{H}$ I gas mass of $8 \times 10^{7} M_{\odot}$. Despite its narrow $\mathrm{H}$ i line width, this galaxy has a rather bright absolute magnitude of $M_{B}=-16.2$. This gives an $M_{\mathrm{dyn}} / L_{B}$ value of approximately 0.4 in solar units.

It is also listed as an uncertain member of the Virgo Cluster in the VCC; however, its velocity of $1655 \mathrm{~km} \mathrm{~s}^{-1}$ (Grogin, Geller, \& Huchra 1998) is consistent with cluster membership. The morphological appearance (Figs. 2 and 3) and quantitative morphology of this galaxy suggest that it is a dwarf elliptical-like object (Table 4), with low asymmetry and concentration values. The clumpiness index $S$ is moderately high, suggesting the presence of some low level of star formation. There are, however, no measured colors for VCC 1713, which if it were very blue, would qualify as a dwarf irregular object. This object is our best candidate for being an object in an ongoing early transition phase between a star-forming and quiescent LMCG.

\subsection{Previous Detections}

The seven following objects, all classified as dE galaxies, were previously detected in $\mathrm{H}$ I. In some cases we also discuss the possibility that some of these objects are not earlytype LMCGs, but are misclassified star-forming galaxies or spurious $\mathrm{H}$ I detections.

\subsubsection{VCC 31}

VCC 31 has a magnitude of $M_{B}=-16.4$ and was detected in $\mathrm{H}$ I using the Arecibo telescope by van Zee, Haynes, \& Giovanelli (1995) and at Nançay by van Driel et al. (2000). Van Zee et al. (1995) determined that this galaxy does not have an extended $\mathrm{H}$ I distribution and has an $M_{\mathrm{H}} /$ $L_{B}$ value high enough to be considered a compact dwarf. Van Driel et al. (2000) also detected this object in $\mathrm{H} \mathrm{I}$, although they find a much higher $W_{20}$ value $\left(312 \mathrm{~km} \mathrm{~s}^{-1}\right)$ than van Zee et al. $\left(1995 ; 132 \mathrm{~km} \mathrm{~s}^{-1}\right)$. This indicates that VCC 31 may have extended gas, since the velocity width and flux of the Nançay observations are much larger than the Arecibo results, which used a smaller 3.5 beam. There are also no nearby galaxies that would have confused the Nançay results, further suggesting that there is extended $\mathrm{H}$ I gas around VCC 31 . This galaxy appears symmetric and dwarf elliptical-like on the DSS and WIYN images (Figs. 2 and 3). Its quantitative morphology (Table 4) is also consistent with a dwarf elliptical classification. Using the van Zee et al. (1995) parameters of the $\mathrm{H}$ I line for this galaxy, we find an $M_{\mathrm{dyn}} / L_{B}$ ratio of $\sim 2.4$ in solar units, while the van Driel et al. (2000) values give ratios a factor of $\sim 5$ higher.

\subsubsection{VCC 168}

VCC 168 was first detected in H I using Arecibo by Hoffman et al. (1987), who classified this object as a dE2 or ImIV galaxy based on the VCC. This object was later classified by Binggeli \& Cameron (1993) as a true dE2 galaxy. It is not easily visible on the DSS images (Fig. 2), but clearly appears as a dwarf elliptical-like object on the WIYN image, with a quantitative morphology consistent with this interpretation (Table 4), including a light profile well fitted by an exponential (Table 4, Fig. 3). Heller et al. (1999) searched for $\mathrm{H} \alpha$ in VCC 168 , but found none. VCC 168 is also symmetric, with little internal structure, based on its image in Heller et al. (1999) and in Figure 3, implying that the dwarf elliptical classification is the correct one. ${ }^{6}$ Almoznino \& Brosch (1998) observed VCC 168 in $B V R$, finding a color $(B-V)$ $=0.76 \pm 0.17$, the reddest $(B-V)$ color in their sample. This suggests an older stellar population, more than 2 Gyr since the cessation of significant star formation. This is the case even if the stars are metal-rich (Worthey 1994); VCC 168 thus appears to be a quiescent LMCG with gas. Its $\mathrm{H}$ I mass of $3 \times 10^{7} M_{\odot}$ is unusually high for such a faint dwarf elliptical galaxy. It also has an $\mathrm{H}$ I line width of $W_{20}=64$ $\mathrm{km} \mathrm{s}^{-1}$, suggesting a dynamical mass-to-light ratio of $M_{\mathrm{dyn}} /$ $L_{B} \sim 2.5$ (Table 3 ).

\subsection{3. $V C C 405$}

VCC 405 is the faintest object in our H I-detected sample, with $B \sim 20$ (VCC), or $M_{B} \sim-11.5$, and it does not appear on DSS images (Fig. 2). This object was marginally detected with Arecibo at $21 \mathrm{~cm}$ (Duprie \& Schneider 1996) with a single observation, and thus the detection could be spurious. Based on their H I spectrum and the very unlikely physical parameters derived from its $\mathrm{H}$ I profile (Table 3), we conclude that this object is indeed probably a false $\mathrm{H}$ i detection and do not consider it in our subsequent analysis.

\subsubsection{VCC 608}

VCC 608 (NGC 4323), classified as a dE4, N in the VCC, is the second brightest Virgo dwarf elliptical galaxy detected in neutral hydrogen, with $M_{B}=-16.6$. It was first detected in H I by Huchtmeier \& Richter (1986). VCC 608 also has a fairly red color, with $(B-V)=0.87 \pm 0.06$ (de Vaucouleurs et al. 1991), consistent with an old stellar population (see the argument in $\S$ 3.5.2). Ryden et al. (1999) determined that this galaxy has a mean ellipticity of 0.39 with isophotal fits giving $\left\langle a_{4} / a\right\rangle$ values consistent with a disky structure. Gavazzi et al. (2001) fitted surface photometry in the nearIR $H$ band $(1.6 \mu \mathrm{m})$, finding a good exponential fit and a low concentration of light, further confirmation that this is morphologically similar to a dwarf galaxy. VCC 608 also has an $M_{\text {dyn }} / L_{B}$ ratio of $\sim 2.5$, based on its $\mathrm{H}_{\mathrm{I}}$ line width. It also contains a high $\mathrm{H}$ I gas content of $\sim 10^{9} M_{\odot}$ and has one of the highest $\mathrm{H}$ I gas mass fractions in our sample (Table 3 ).

\subsection{5. $V C C 797$}

VCC 797, with $W_{50}=28 \mathrm{~km} \mathrm{~s}^{-1}$, has the narrowest H I line of any galaxy in our sample and the smallest optical scale length of $0.24 \mathrm{kpc}$. The internal velocity dispersion is low, indicating that its observed $\mathrm{H}$ I velocity width may be dominated by turbulence. It is also the only low-mass galaxy that is near a giant galaxy, in this case about $3^{\prime}$ south of M85 (Fig. 2). H I in VCC 797 was discovered by Burstein,

\footnotetext{
${ }^{6}$ Heller et al. (1999) only find 5 of 43 objects without $\mathrm{H} \alpha$ emission in their survey of Virgo Cluster irregular galaxies, one of which is VCC 168. This further suggests that this object is not a true irregular galaxy. The irregular classification of VCC 168 by Heller et al. is solely based on its $\mathrm{H} \mathrm{I}$ detection by Hoffman et al. (1987)
} 
Krumm, \& Salpeter (1987), who detected it during $21 \mathrm{~cm}$ observations of M85. Because of the presence of $\mathrm{H} \mathrm{I}$, Burstein et al. (1987) considered VCC 797 to be a dwarf irregular galaxy, although it is classified as a dE3, $\mathrm{N}$ galaxy in the VCC. The VCC classification is based on higher resolution images than those available to Burstein et al. (1987). Burstein et al. (1987), however, only marginally detect the object at a $4 \sigma$ level of significance. We tentatively consider this to be an $\mathrm{H}$ I-detected dE galaxy and not a spurious signal, although this should be confirmed by observing it again at $21 \mathrm{~cm}$ with higher sensitivity.

\subsubsection{VCC 1949}

VCC 1949 is the brightest galaxy $\left(M_{B}=-17.1\right)$ in our sample, and it appears as an early-type dwarf on DSS images (Fig. 2). It was classified as a $\mathrm{dSBO}(4), \mathrm{N}$ or $\mathrm{dE}(6,4)$, $\mathrm{N}$ galaxy in the $\mathrm{VCC}^{7}$ It was first detected in $\mathrm{H}$ i by Huchtmeier \& Richter (1986) and later reobserved at $21 \mathrm{~cm}$ by Haynes \& Giovanelli (1986). The optical image of this galaxy is well fitted by an exponential profile (Binggeli \& Cameron 1993). VCC 1949 is one of three galaxies in the present sample with very large $\left(>250 \mathrm{~km} \mathrm{~s}^{-1}\right)$ inferred $\mathrm{H} \mathrm{I}_{\mathrm{I}}$ velocity widths (Table 3 ), giving it a high measured dynamical mass-to-light ratio of $M_{\mathrm{dyn}} / L_{B} \sim 20$ in solar units. There are no obvious companions that could produce this large line width from confusion.

\subsubsection{VCC 2062}

VCC 2062 has been studied previously in H I by both Hoffman et al. (1993) and van Driel \& van Woerden (1989). It is about $2 \mathrm{kpc}(0.4)$ away from the irregular galaxy NGC 4694, with which it may be interacting. VCC 2062 was classified as a dE galaxy in the VCC, although the DSS image in Hoffman et al. (1993) suggests that this classification is incorrect and that the galaxy is more likely an irregular galaxy. Hoffmann et al. also found very blue colors for this object, with $(B-V)=0.35$ and $(V-R)=0.19$. These colors indicate that star formation has recently occurred, since an extremely metal-poor stellar population, which can also produce blue colors, would have to be less than $700 \mathrm{Myr}$ old to produce these colors (Worthey 1994). The presence of

\footnotetext{
${ }^{7}$ Binggeli \& Cameron (1993) list VCC 1949 as a dS0 galaxy.
}

young stars reveals that this object is probably not a classical dE galaxy (i.e., old stars and no gas), despite its classification in the VCC.

Because if its fairly high $M_{\mathrm{H}} / L_{B}$ ratio of $\sim 55$, this object is also by far the most gas-rich member of our sample. The high $M_{\mathrm{H}} / L_{B}$ ratio exceeds that of typical values found for blue compact dwarf (van Zee et al. 1995) and irregular galaxies (see, e.g., Roberts \& Haynes 1994), another indication that it may be an interacting system.

In summary, we conclude that seven of the nine detections of $\mathrm{H}$ I in LMCGs are genuine and reject as spurious the H I dE detection claims for VCC 405 and VCC 2062.

\section{INTERPRETATIONS}

\subsection{Incorrect Morphological Identifications}

Although the galaxies discussed in this paper are classified as dE galaxies in the VCC, other studies have given different classifications for a few of these objects, mostly the dwarf irregular class. Could all of these $\mathrm{H}$ I-detected LMCGs in our sample be misclassified dwarf irregular galaxies? To some degree, the problem is semantic, assuming that the VCC used a consistent classification approach throughout the Virgo Cluster. The identification in the VCC of a $\mathrm{dE}$ galaxy therefore may be biased, but as long as this bias is consistently applied throughout, then the VCCclassified "dE galaxies" should represent a homogeneous population equally distributed across the cluster.

We also reject the incorrect morphological identification explanation for the following reasons. The qualitative and quantitative morphologies for these objects, as seen in the DSS frames and in the WIYN images, suggest quiescent systems, with no obvious evidence for star formation, with the possible exception of VCC 1713. The available colors for VCC 168 (§ 3.5.2) and VCC 608 (§ 3.5.4) also demonstrate that some H I-detected early-type LMCGs potentially have older dE-like stellar populations. The H I gas contents for these systems are also too low for these objects to be misclassified normal dwarf irregular galaxies, since Virgo dIrr galaxies have higher $\mathrm{H}$ I gas masses and fluxes than those listed in Table 3 (see, e.g., Hoffman et al. 1987; Gallagher \& Hunter 1989; see also Table 5). Therefore, we conclude that these $\mathrm{H}$ I-detected LMCGs are probably not misclassified

TABLE 5

Averages Properties and Dispersions for Different Populations

\begin{tabular}{|c|c|c|c|c|}
\hline Population & $\left\langle M_{B}\right\rangle$ & $\begin{array}{c}\left\langle M_{\mathrm{H}_{\mathrm{I}}}\right\rangle \\
\left(\times 10^{9} M_{\odot}\right)\end{array}$ & $\left\langle f_{\text {gas }}\right\rangle^{\mathrm{a}}$ & $\begin{array}{c}\left\langle M_{\mathrm{H}_{\mathrm{I}}} / L_{B}\right\rangle \\
\left(M_{\odot} / L_{\odot}\right)\end{array}$ \\
\hline $\mathrm{H}_{\mathrm{I}} \mathrm{LMCG}_{\mathrm{s}}{ }^{\mathrm{b}} \ldots$ & $-15.4 \pm 1.2$ & $0.28 \pm 0.44$ & $0.30 \pm 0.28$ & $0.57 \pm 0.57$ \\
\hline Virgo Irr' ${ }^{\mathrm{c}}$ & $-16.4 \pm 0.8$ & $0.52 \pm 0.98$ & & $0.61 \pm 0.54$ \\
\hline Virgo Amorphous ${ }^{c}$............ & $-17.4 \pm 1.0$ & $0.12 \pm 0.13$ & & $0.07 \pm 0.05$ \\
\hline LG Irr ${ }^{d}$ & $-14.5 \pm 2.7$ & $0.20 \pm 0.26$ & $0.30 \pm 0.24$ & $1.4 \pm 1.7$ \\
\hline LG dE ${ }^{\mathrm{d}}$ & $-13.7 \pm 2.2$ & $(3 \pm 6) \times 10^{-4}$ & $(1.5 \pm 4) \times 10^{-3}$ & $0.001 \pm 0.002$ \\
\hline LG TT $^{\mathrm{e}}$ & $-10.8 \pm 1.0$ & $(2 \pm 2) \times 10^{-3}$ & $0.15 \pm 0.21$ & $0.44 \pm 0.28$ \\
\hline LG Spiral ${ }^{\mathrm{d}}$. & $-19.3 \pm 0.9$ & $4.2 \pm 2.3$ & $0.11 \pm 0.11$ & $0.54 \pm 0.38$ \\
\hline
\end{tabular}

a Defined, as in Table 3, as the fraction of the total mass contained in $\mathrm{H}$ I gas, where the total mass is approximated by $M_{\text {dyn }}$.

${ }^{\mathrm{b}}$ From the data presented in this paper, especially Table 3.

${ }^{c}$ Based on the observational data presented in Gallagher \& Hunter 1989.

d From data in van den Bergh 2000 and Mateo 1998.

e Transition types (TT) include the following galaxies: Antila, Aquarium (DDO 210), LGS-3 (Pisces), Phoenix, Pegasus, DDO 155, and DDO 216. The data are from van den Bergh 2000 and Mateo 1998. 


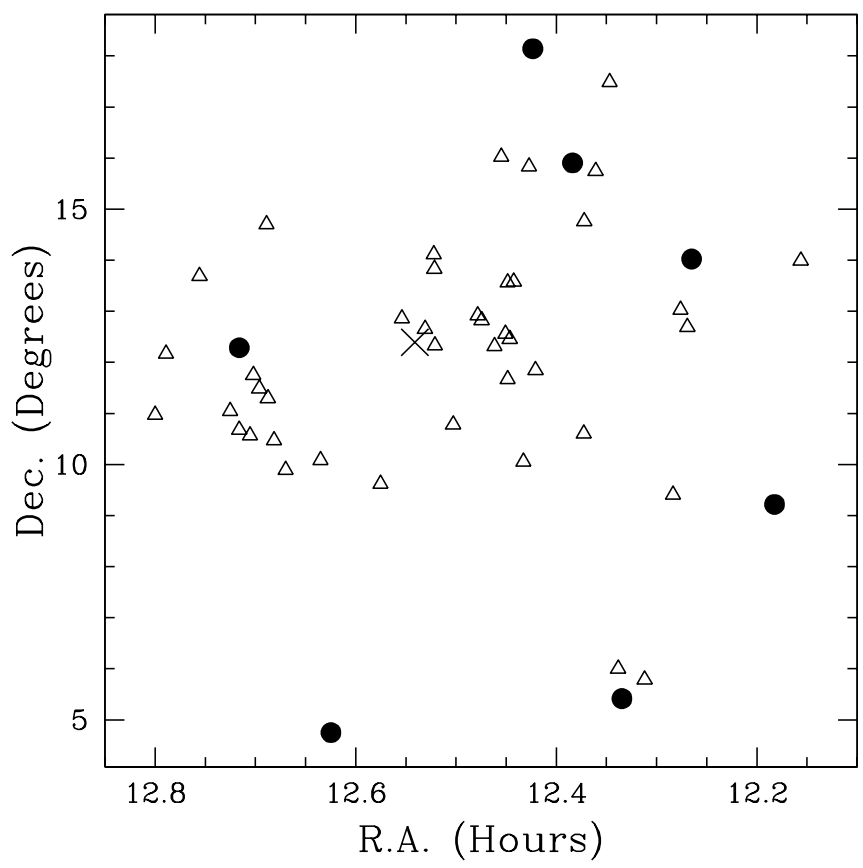

FIG. 5.-Distribution on the sky of $\mathrm{dE}$ galaxies observed at $21 \mathrm{~cm}$ in the Virgo Cluster. The open triangles represent nondetections, while the filled circles show the locations of the $\mathrm{dE}$ galaxies with detected $\mathrm{H}$ I. The cross toward the center shows the location of the giant elliptical M87.

dwarf irregular galaxies, with the one exception being VCC 2062 , which is not included in the following analyses.

\subsection{Patterns of $\mathrm{H}$ I Depletion and Orbital Structure}

In Figures 5 and 6 we show the spatial pattern of $\mathrm{H} \mathrm{I}$ depletion of Virgo classified dwarf elliptical galaxies; in Figure 5 galaxies with $\mathrm{H}$ i detections are plotted as large filled circles and non-H I detections as open triangles. All of the

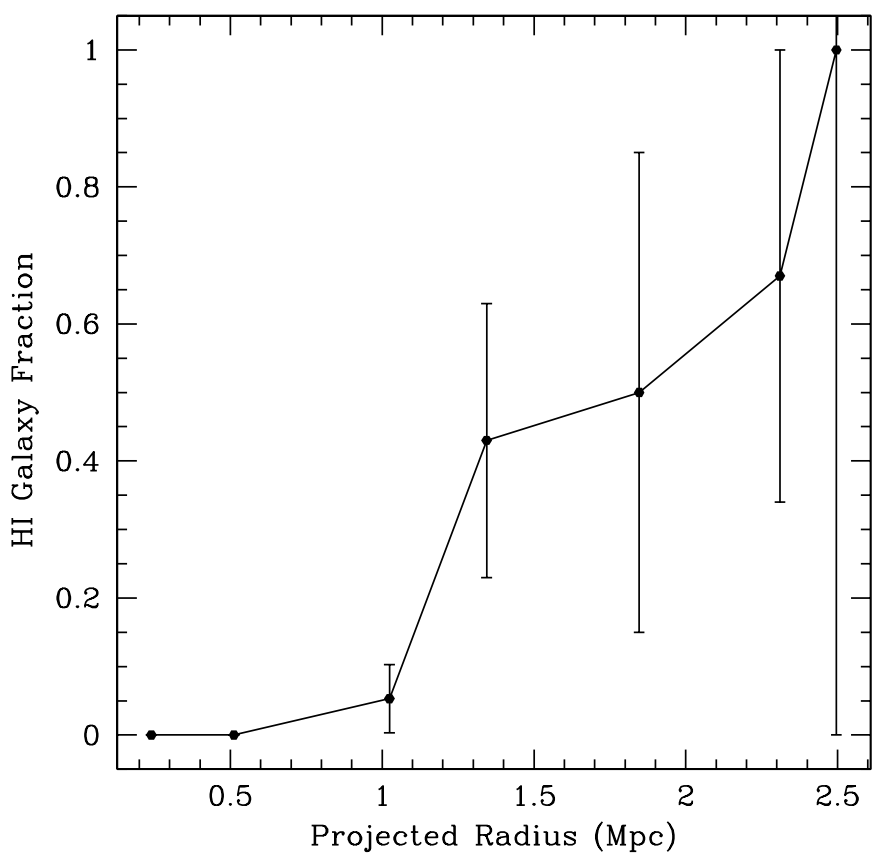

FIG. 6.-Fraction of Virgo classified dwarf elliptical galaxies detected in $\mathrm{H}$ I as a function of projected distance from the center of the cluster.
LMCGs classified as $\mathrm{dE}$ galaxies with $\mathrm{H}$ I detections are projected toward the outer parts of the cluster. This is quantitatively shown in Figure 6, which plots the fraction of LMCGs classified as $\mathrm{dE}$ galaxies with $\mathrm{H}$ I detections as a function of projected distance from the luminosity-weighted center of the Virgo Cluster, as defined by Sandage, Binggeli, \& Tammann (1985). Within a projected distance of $0.5 \mathrm{Mpc}$ of the center, the lower limit to the true three-dimensional distance, we find no galaxies classified as $\mathrm{dE}$ with $\mathrm{H}$ I detections.

The presence of most of the H I LMCGs in the outer parts of Virgo may help reveal the origin of these objects. One obvious explanation is that these galaxies have just been accreted into the cluster and have not yet crossed the core. The other possibility is that these galaxies have orbits that take them through the core of the cluster and somehow retain or replenish their $\mathrm{H}$ I gas. For the purposes of argument, we discuss the infall case and two idealized orbiting scenarios: radial and high angular momentum orbits.

Cosmological models of galaxy clusters show that most cluster members are originally on moderate, or low, angular momentum orbits (see, e.g., Crone, Evrard, \& Richstone 1994; Ghigna et al. 1998; Huss, Jain, \& Steinmetz 1999). Gas-stripping processes are also most effective for galaxies on nearly radial orbits (Vollmer et al. 2001). The survival of $\mathrm{H}$ I in low-mass cluster galaxies therefore depends on orbital parameters, such that galaxies in more circular orbits that avoid the core are more likely to retain their $\mathrm{H}$ I. Signatures of this effect also may be seen in samples of giant galaxies (see, e.g., Dressler 1986; Solanes et al. 2001) and might also be present in some LMCGs. However, Solanes et al. (2001) do not find clear evidence for this behavior in Virgo, possibly as a result of complications associated with interactions with substructure in this cluster (see also Vollmer et al. 2001).

Evidence that our H I LMCGs are on high angular momentum orbits includes the distribution of their radial velocities. The expected signature for galaxies on high angular momentum orbits would be a flat-topped distribution in velocities. Galaxies on radial orbits would have a Gaussian distribution peaked at the mean velocity of the cluster. The average velocity of the $\mathrm{H}$ I LMCGs is relatively large and not centered at the average velocity of the cluster, and the distribution of velocities is nonuniform, different from expectations of both the radial and high angular momentum distribution. Although our distribution is not flat-topped, it is clearly not uniformly distributed about the mean cluster velocity. The velocity distribution of the $\mathrm{H}$ I-rich early-type LMCG velocity distribution is shown in Figure 7, where the shaded part displays the distribution of the $\mathrm{H}$ I-detected LMCGs and the open histograms are the velocity distribution of the nondetections. Although we are in the regime of small-number statistics, we can make some arguments based on the data available.

The average velocity of the non-H I-detected objects is $1287 \pm 183 \mathrm{~km} \mathrm{~s}^{-1}$, and the $\mathrm{H}$ I-detected objects have an average velocity of $1669 \pm 553 \mathrm{~km} \mathrm{~s}^{-1}$. The velocity dispersions are very similar, with $\sigma=685$ and $697 \mathrm{~km} \mathrm{~s}^{-1}$ for the nondetected and detected objects, respectively. However, for galaxies at more than $1.5 \mathrm{Mpc}$ projected separation from the center of Virgo, the velocity dispersion for the $\mathrm{H} \mathrm{I}$ LMCGs is $200 \mathrm{~km} \mathrm{~s}^{-1}$ higher than for the LMCGs with non-H I detections. A Kolmogorov-Smirnov test reveals a $24 \%$ probability that these two distributions are similar. 


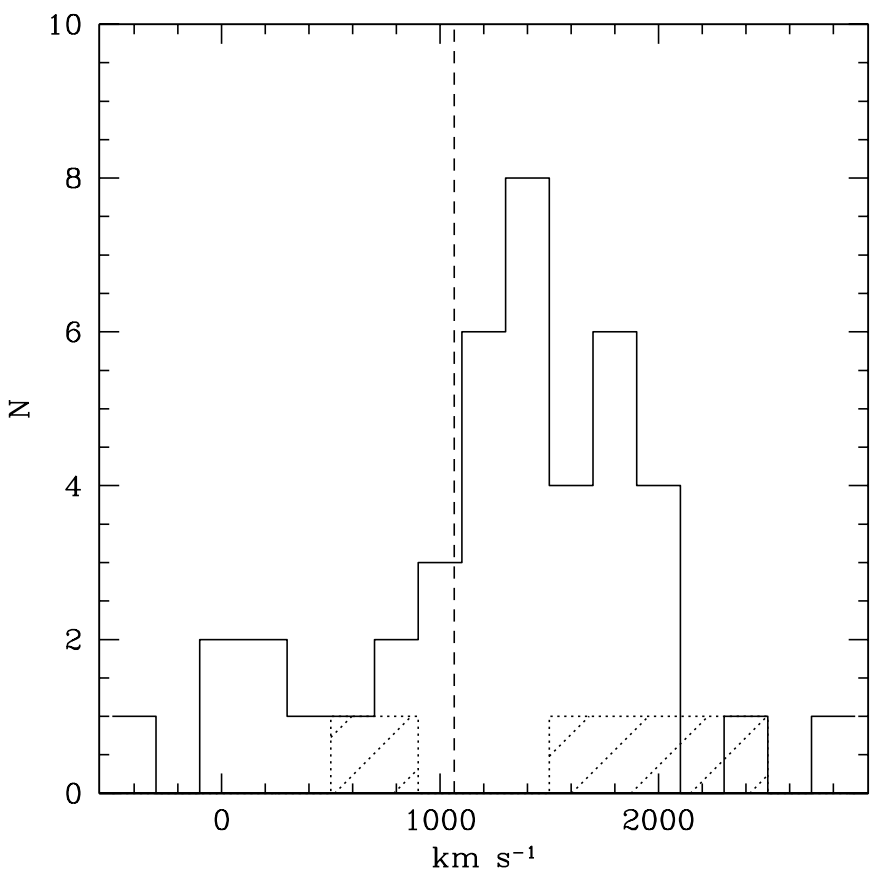

FIG. 7.- Histogram of radial velocities for $\mathrm{dE}$ galaxies searched for in $\mathrm{H}$ I. The open histograms represent the dE nondetections, while the shaded histogram shows Virgo dE galaxies with H I detections. The vertical dashed line shows the mean velocity of the Virgo Cluster, from Paper I.

Figure 8 shows the magnitudes for the H I-detected (shaded histogram) and nondetected (open histogram) objects, demonstrating that unlike the radial velocities, the magnitudes of the detected objects are not significantly skewed toward any particular values, although they are on average slightly fainter.

To determine the significance of the $\sim 400 \mathrm{~km} \mathrm{~s}^{-1}$ difference in average velocity between the $\mathrm{H}$ I-detected and non-

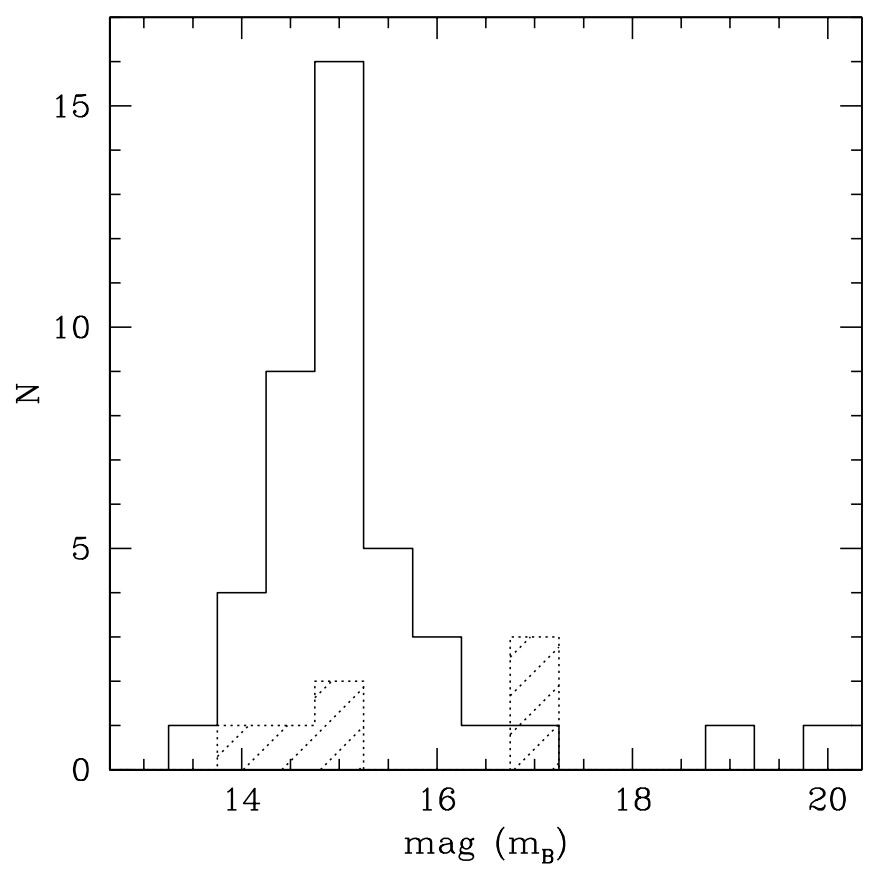

FIG. 8.-Histograms of apparent $B$-band magnitudes for nondetected (open histograms) and $\mathrm{H} \mathrm{I}$-detected (shaded histograms) Virgo Cluster dE galaxies. The shadings are the same as in Fig. 7. detected LMCGs, we perform a Monte Carlo simulation based on the velocities of $\mathrm{dE}$ galaxies in Virgo (Paper I). Using the $\sigma$ and the mean velocity of dE galaxies from Paper I, we randomly select seven galaxy velocities from this Gaussian distribution and compute the mean of these seven values. This exercise reveals less than a $1 \%$ chance $(\sim 3 \sigma)$ that the $400 \mathrm{~km} \mathrm{~s}^{-1}$ difference is random. Therefore, the fact that the velocities of these $\mathrm{H}$ I-detected LMCGs are not near the mean cluster velocity of $1064 \pm 34 \mathrm{~km} \mathrm{~s}^{-1}$ and are found in the outer parts of Virgo is an indication that a fraction of these H I LMCGs are not on radial orbits and are likely on high angular momentum ones with some velocity substructure induced, perhaps by galaxies infalling within groups.

\subsection{Gas Stripping, Replenishment, and Star Formation}

The spatial pattern of LMCGs with detectable H I suggests that gas stripping has occurred through interactions with the intracluster medium (ICM; Dressler 1986; Vollmer et al. 2001). Such stripping can happen in a number of ways. The most commonly suggested method is ram pressure stripping, which can deplete a dwarf galaxy rapidly in the inner regions of a cluster, in typically less than $0.1 \mathrm{Gyr}$ (Mori \& Burkert 2000) for a cold dark matter-like (CDMlike) halo, although this depends on the time for shock waves to traverse the galaxy, the form of the galaxy's gravitational potential, and properties of the ICM. A more gradual mass loss may also occur because of Kelvin-Helmholtz instabilities (Nulsen 1982), although our estimate below suggests that it is not likely important for Virgo Cluster dwarf galaxies.

\subsubsection{Ram Pressure Stripping}

The ram pressure on a galaxy from the interaction of intracluster gas is given by the equation $P=\rho_{\mathrm{ICM}} v_{\perp}^{2}$, where $v_{\perp}$ is the velocity perpendicular to the plane of a galaxy. The efficiency of gas stripping also depends on the gravitational potential $\phi$ of a galaxy, i.e., its ability to hold onto its material. The vertical gravitational acceleration of interstellar medium (ISM) clouds in a disk galaxy is given by $\partial \phi / \partial z$. If we define a galaxy's gas surface density as $\sigma$, then ram pressure stripping occurs in the galaxy when

$$
\frac{\partial \phi}{\partial z} \sigma<\rho_{\mathrm{ICM}} v_{\perp}^{2}
$$

Here $\rho_{\text {ICM }}$ is the density of the ICM of the Virgo Cluster and $v_{\perp}$, as before, is the relative velocity perpendicular to the plane of the disk. This formula, first proposed by Gunn \& Gott (1972), is found to be a good approximation based on detailed simulations (see, e.g., Abadi, Moore, \& Bower 1999; Vollmer et al. 2001).

To determine the ICM density $n(r)$ as a function of radius, we use a $\beta$-model (Cavaliere \& Fusco-Femiano 1976) and the parameters for the Virgo Cluster given by Vollmer et al. (2001),

$$
n(r)=n_{0}\left(1+\frac{r^{2}}{r_{0}^{2}}\right)^{-(3 / 2) \beta} .
$$

In this equation $r$ is the physical distance from the center of the cluster, $n_{0}$ is the central ICM gas density, which is $n_{0}=4 \times 10^{-2} \mathrm{~cm}^{-3}, r_{0}=13.4 \mathrm{kpc}$, and $\beta=0.5$ for Virgo intracluster gas (Vollmer et al. 2001). By knowing the gas 


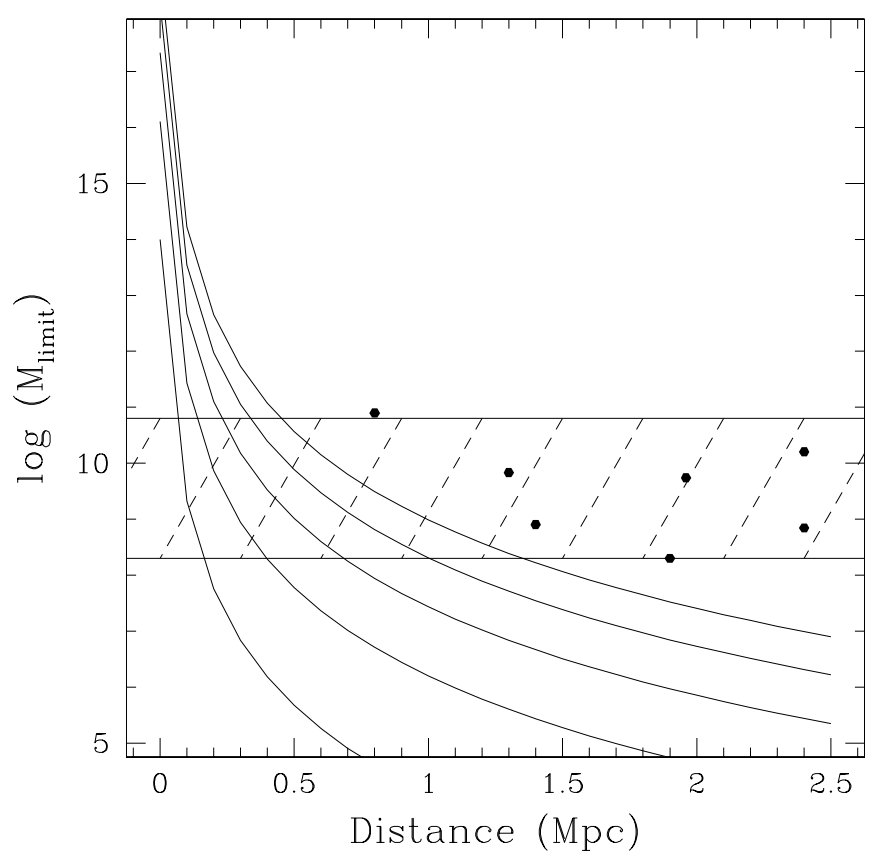

FIG. 9.-Minimum galaxy mass $M_{\text {limit }}$, above which $\mathrm{H}$ I gas is expected to survive ram pressure stripping, as a function of distance from the Virgo Cluster center. The five solid lines show this relationship for galaxy orbital velocities relative to the ICM of $v=250,500,750,1000$, and $1250 \mathrm{~km} \mathrm{~s}^{-1}$ at increasing mass limits. The filled circles show the projected locations of the seven $\mathrm{H}$ I LMCGs studied in this paper, which are all above the minimum mass limits. The hatched region shows the range of total masses for our $\mathrm{H}$ I LMCGs, and dwarf galaxies in general.

density as a function of radius, the fraction of a galaxy's mass in the gas phase, and the velocity of the gas with respect to the ICM, the minimum mass $M_{\text {limit }}$ a galaxy must have to avoid being stripped of gas by ram pressure can be computed analytically (Mori \& Burkert 2000) for CDM halos. If we assume a gas mass fraction of $\sim 0.1-0.3$ for a typical LMCG, we can compute $M_{\text {limit }}$ as a function of $v_{\perp}$ and $n(r)$. Using equation (4) for the gas density as a function of radius in Virgo, we plot in Figure 9 the mass limit $M_{\text {limit }}$ as a function of radius at velocities $v_{\perp}$ from 250 to 1250 $\mathrm{km} \mathrm{s}^{-1}$. Also plotted on this diagram, as filled circles, are the dynamical masses and projected distances from the cluster center for each of the seven LMCGs with confident H I detections. This figure shows that at the relatively large distances of the detected H I LMCGs from the Virgo center, their masses are high enough such that ram pressure stripping cannot rapidly deplete them of all their gas. Thus, we see that high angular momentum orbits in the outer parts of the Virgo Cluster could allow slow gas removal from accreted field galaxies.

\subsubsection{Kelvin-Helmholtz Instabilities}

The interface between the Virgo ICM and the ISM of member galaxies produces a Kelvin-Helmholtz instability that could gradually extract gas from these galaxies, aiding ram pressure in removing gas. The lower mass limit for Kelvin-Helmholtz instabilities to be efficient is roughly $10^{3}$ times higher than the ram pressure stripping limit (Mori \& Burkert 2000). It is, however, unlikely that gas-rich LMCGs are being depleted gradually through this process. The mass-loss rate induced by Kelvin-Helmholtz instabilities is given by Nulsen (1982) as

$$
\dot{M}=\pi R^{2} n(r) v
$$

where $R$ is the radius of the galaxy, $v$ is the velocity of the galaxy relative to the ICM, and $n(r)$ is the mass density of the ICM. Using typical values (Table 3 ) of $R=1 \mathrm{kpc}$ and $v=500 \mathrm{~km} \mathrm{~s}^{-1}$, we find that $\dot{M} \sim 10^{3} M_{\odot} \mathrm{Myr}^{-1}$. The timescale for gas depletion of a typical LMCG via KelvinHelmholtz instabilities is then $\sim 10-15$ Gyr.

If a small galaxy entered the Virgo Cluster sometime in the last few Gyr on a high angular momentum orbit, then it would not yet have been stripped of all its gas, either through ram pressure or Kelvin-Helmholtz instabilities. Both of these processes are occurring, but they are slowly removing gas from galaxies on high angular momentum orbits in the outer parts of the Virgo Cluster. On the other hand, it appears that these galaxies would have been completely stripped of all their gas if they had existed within the cluster on the order of a Hubble time, no matter what their orbits are. The above arguments again suggest that these $\mathrm{H}$ I LMCGs are objects that must have entered the cluster in the last few Gyr, or have just been accreted.

\subsubsection{Gas Replenishment}

During the normal course of stellar evolution, stars in galaxies will deposit gas into the ISM, thereby increasing the presence of H I. Gas is returned to the ISM from mass loss in stars during the asymptotic giant branch and planetary nebula phases. The amounts returned vary, depending on the age and total mass of the stellar populations (see, e.g., Faber \& Gallagher 1976). Following the arguments in Faber \& Gallagher (1976) for giant elliptical galaxies and in Gnedin et al. (2002) for the globular cluster $\omega$ Centauri, we calculate that the mass returned to the ISM for an LMCG with a stellar mass of $\sim 10^{8} M_{\odot}$ is $\sim 10^{6} M_{\odot} \mathrm{Gyr}^{-1}$. If we assume that the $\mathrm{H}$ I LMCGs have been in the cluster for a few Gyr, then not enough time has elapsed to produce the observed $\mathrm{H}$ I gas content of the $\mathrm{H}$ I-detected early-type LMCGs from evolved stars. However, if this was the complete story, we might expect substantial $\mathrm{H}$ i content to be a common feature of very old early-type LMCGs, but we see very little $\mathrm{H}$ I in field elliptical galaxies or in LMCGs (see, e.g., Knapp, Kerr, \& Bowers 1978; Young \& Lo 1997; Sage, Welch, \& Mitchell 1998). It is still not known with certainty why giant elliptical galaxies are depleted of cold interstellar gas, but whatever the cause of depletion, it is likely occurring in LMCGs as well. In summary, we do not consider the return of gas from stellar evolution a likely scenario to explain the $\mathrm{H}$ I gas content of these H I LMCGs.

\subsubsection{Impulsive Encounters: Dynamical Stripping}

The high number density and large relative velocities of cluster galaxies make impulsive encounters between them common and can result in mass loss, such as in the harassment scenario (see, e.g., Moore, Lake, \& Katz 1998), although this effect is likely not important for the H I LMCGs since they are located in the outer parts of Virgo. The energy imparted to a galaxy with mass $M_{\text {gal }}$ during an impulsive encounter between two spherical galaxies is proportional to $E \propto M_{\text {pert }} M_{\text {gal }} b^{-4} V^{-2}$ (Spitzer 1958), where $M_{\text {pert }}$ is the mass of the perturbing galaxy, $b$ is the distance between the two galaxies, and $V$ is the relative velocity between them. The large separation of the H I LMCGs from the center of 
Virgo is an indication that the factor $b$ is large for most cluster galaxies. At a typical LMCG impact parameter of $0.5-1$ $\mathrm{Mpc}$, the energy imparted to a galaxy and its resulting mass loss due to impulsive encounters are very small (Aguilar \& White 1985). The strongest effects of mass loss involve cluster galaxies on nearly radial orbits, where the parameter $b$ becomes small (Moore et al. 1998). If the H I LMCGs are on high angular momentum orbits, as has been argued, then they do not feel the strong effects of impulsive encounters and thus will not lose a significant amount of mass from these interactions. This is especially true if the H I LMCGs have only been in the cluster for a short time. We conclude, therefore, that the only processes strongly affecting the $\mathrm{H}$ I LMCGs are ram pressure-induced gas loss.

\subsection{Morphological Transformations}

The question we address in this section is whether or not the H I-detected LMCGs have recently been accreted into Virgo (and not yet been stripped of their gas) or if they have been in the cluster for at least a few Gyr, having evolved from a precursor of different morphology. As we argued in $\S \S 4.2$ and 4.3, it is unlikely that these galaxies are an old cluster population. Here we conclude that these LMCGs originate from accreted field populations.

The idea that galaxies can undergo a morphological transformation in clusters has considerable observational support. Distant clusters contain large populations of blue, distorted galaxies (see, e.g., Oemler, Dressler, \& Butcher 1997; Couch et al. 1998), which are not seen in the same proportion in nearby clusters (cf. Conselice \& Gallagher 1998, 1999). There is also evidence that S0 galaxies are less common in moderate-redshift clusters than in comparison to similar clusters at low redshifts (Dressler et al. 1997). Furthermore, in Paper I we argue that all galaxy types, except giant elliptical galaxies, have kinematic and spatial properties suggesting that they were accreted into Virgo after its initial formation, perhaps because of hierarchical accretion into the cluster (Kauffmann 1995). Once they enter the cluster, galaxies can morphologically evolve into earlier types (Moore et al. 1998; Mao \& Mo 1998; Abadi et al. 1999; Quilis, Moore, \& Bower 2000). What is missing from these arguments is whether or not galaxies in nearby clusters are undergoing evolution, or morphological transformations, as they should if any are still being accreted into clusters, albeit at reduced rates compared to clusters at high redshift (see, e.g., Tully \& Shaya 1984). Previous observations suggest that there are some small galaxies in the Virgo Cluster that appear to be undergoing rapid evolution (Gallagher \& Hunter 1989; Vigroux et al. 1986), but the extent of this process is currently unknown. Does most active evolution of cluster galaxies, including morphological transformations, occur only in the distant past? We argue here that $\mathrm{H} \mathrm{I}$ LMCGs are possible examples of objects slowly undergoing transformations today. The evidence, which we discuss in detail below, includes the outer spatial distribution of these detections in Virgo, their mixed morphological appearances, their gas and stellar content, and the correlation between $\mathrm{H}$ I line width and total $B$-band magnitude.

\subsubsection{Morphologies and Stellar Populations: Dwarf Elliptical-like}

We can try to understand when and how stars in $\mathrm{H} \mathrm{I-}$ detected LMCGs formed by examining their morphologies and the limited spectral information we have. The morphol- ogies and structural parameters of these galaxies (Figs. 2 and 3, Table 4) are clearly not influenced by star formation. The red colors of VCC 168 and VCC 608 demonstrate that these objects contain older stellar populations, and the clumpiness parameter $S$ is low enough that any star formation in these H I LMCGs must be smoothed out. The question is, how long does it take for a young stellar population to become this red and smooth? A galaxy's stellar population will be smoothed out after approximately a crossing time when young stars die and the population dissolves into the background (Harris et al. 2001), which takes a few Gyr for these galaxies, especially if their random motions are increased slightly through dynamical heating (Moore et al. 1998). For a stellar population to become as red as VCC 168 and VCC 608 from a blue star-forming irregular or disk galaxy requires roughly the same amount of time. These $\mathrm{H}$ I-detected LMCGs therefore have stellar population and morphological characteristics of dwarf elliptical galaxies and must have had their last major episode of star formation several Gyr ago.

\subsubsection{Gas Content: Dwarf Irregular-like}

A diagnostic for understanding the origin of $\mathrm{H}$ I-detected LMCGs is to compare their optical and $\mathrm{H}$ I properties, including $M_{\mathrm{H}} / L_{B}$ values, absolute magnitudes, and $\mathrm{H}$ I masses, to those of other galaxy populations, including the Gallagher \& Hunter (1989) Virgo Cluster dwarf irregular and amorphous samples. Table 5 lists the averages of several of these quantities as a function of galaxy type. The $\mathrm{H}$ i gas mass fractions and $M_{\mathrm{H}} / L_{B}$ ratios are fairly similar in the various galaxy populations, except for the Local Group dE galaxies, where these values are significantly lower. Dwarf irregular galaxies in the Local Group have the highest $f_{\text {gas }}$ and $M_{\mathrm{H}} / L_{B}$ ratios, while the Local Group dE galaxies have the lowest.

For example, the gas mass fractions $f_{\text {gas }}$ for dwarf irregular galaxies in the Local Group are 0.07, 0.08, 0.2, 0.65, and 0.46 for the Large Magellanic Cloud, NGC 6822, IC 10, IC 1613 , and DDO 221, respectively. The average $f_{\text {gas }}$ value for all Local Group dwarf irregular galaxies is $0.30 \pm 0.24$ (Table 5). Virgo LMCGs classified as dE galaxies with $\mathrm{H} \mathrm{I}$ detections in Virgo have an average $f_{\text {gas }}$ value of $0.30 \pm 0.28$ (Table 3). On the other hand, the gas mass fractions for Local Group dwarf spheroidal galaxies are often less than $10^{-4}$ to $10^{-5} \sim 0$ (Johnson \& Gottesman 1983; Koribalski, Johnston, \& Ortupcek 1994; Young \& Lo 1997; Young 1999).

Some $M_{\mathrm{H}} / L_{B}$ values for the $\mathrm{H}$ I-detected LMCGs are as high or higher than those of some spiral and irregular galaxies, although some gas-rich low-luminosity early types have been found (Sadler et al. 2000). Even if some gas mass is lost through various stripping effects, the fading of stellar populations due to the truncation of star formation ( $\S 4.4 .4)$ may be able to reproduce this. Stellar populations with initial colors similar to those of late-type spiral galaxies and irregular galaxies will become $\sim 1.5$ mag fainter in $\sim 3 \mathrm{Gyr}$, easily allowing for high $M_{\mathrm{H}} / L_{B}$ values even if some gas is removed. Some of these systems could also be low- $M_{\mathrm{H}}$ accreted field galaxies, since examples are known to exist in the field (Knezek, Sembach, \& Gallagher 1999).

Average values of other properties, listed in Table 5, show that Local Group dwarf elliptical/spheroidal galaxies have very different derived $\mathrm{H}$ I gas properties from the Virgo $\mathrm{H}_{\mathrm{I}}-$ 
detected LMCGs classified as $\mathrm{dE}$ galaxies. In fact, the average values are different at a $7 \sigma$ significance. Based on this, the $\mathrm{H}$ I dE galaxies cannot simply be recently accreted analogs of Local Group dwarf elliptical/spheroidal galaxies, since they have too much $\mathrm{H}$ I. Although the $\mathrm{H}$ I-detected LMCGs studied here morphologically appear to be dwarf elliptical galaxies, they could not have originated from $\mathrm{dE}$ galaxies in groups, since no pure dwarf elliptical galaxy has a gas content as large as these LMCGs have. We propose that the only way these galaxies could contain such a high $\mathrm{H}$ I gas mass is for them to have originally been star-forming systems that morphologically evolved into a dE-like object over the last few Gyr through slow gas removal and passive evolution. The most similar galaxy types to the Virgo H Idetected LMCGs are the Local Group transition types, Phoenix and Pisces, and irregular galaxies, which have $f_{\text {gas }}$ and $M_{\mathrm{H}} / L_{B}$ values within $1 \sigma$ of the LMCG values (Fig. 10), although the $\mathrm{H} \mathrm{I}$ masses for these LMCGs are more similar to the values found for the irregular galaxies. As we argue in $\S 4.4 .4$, star formation in these systems could be suppressed.

\subsubsection{Parameter Space: Transition Types}

We can further use diagnostic plots such as Figures 10-13 to understand which of the well-understood nearby galaxy populations is most similar to the $\mathrm{H}$ I LMCGs. Figure 10 plots $f_{\text {gas }}$ and $M_{\mathrm{H}} / L_{B}$ values for individual Local Group spiral galaxies (open squares), irregular galaxies (open triangles), dE galaxies (filled triangles), transition types (crosses), and the Virgo H I LMCGs studied in this paper (filled circles). The average $f_{\text {gas }}$ and $M_{\mathrm{H}} / L_{B}$ values for each type are labeled by a circled small version of their respective identification symbol, and the average values are listed in Table 5. Again, from this figure it appears that the $\mathrm{H} \mathrm{I}$-detected

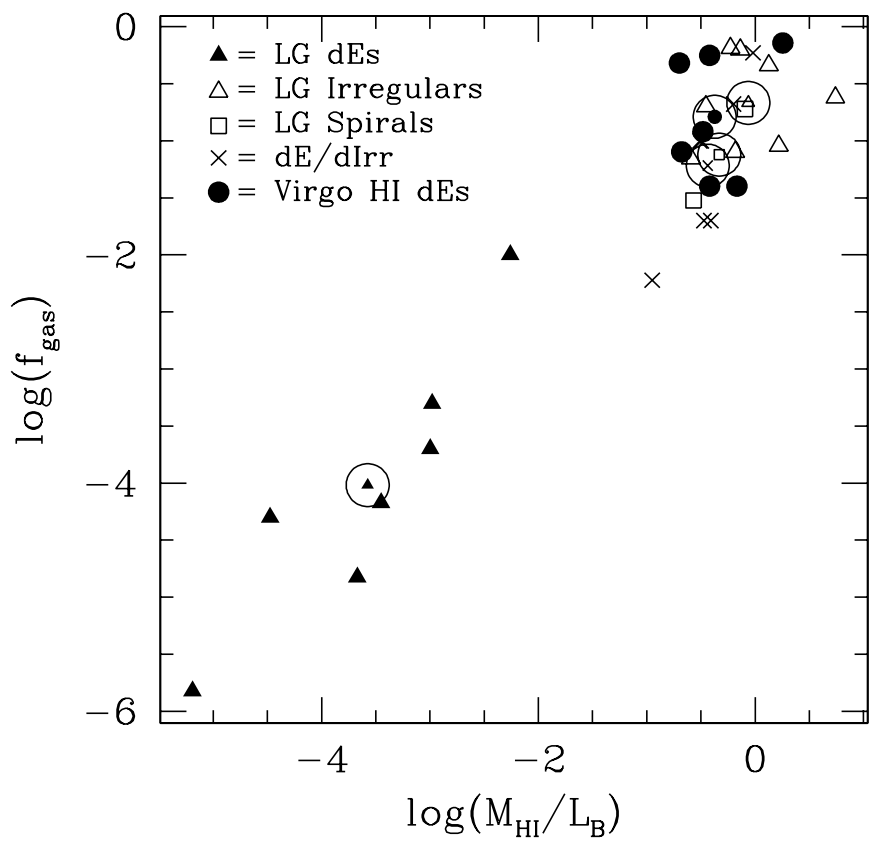

Fig. 10.-Fraction of mass in $\mathrm{H} \mathrm{I}\left(f_{\text {gas }}\right)$ for various Virgo Cluster member galaxy types plotted as function of $M_{\mathrm{H}_{\mathrm{I}}} / L_{B}$. Each separate galaxy population is designated by the labeled symbols. The average position for each type is plotted as a small version of its respective symbol in a circle.

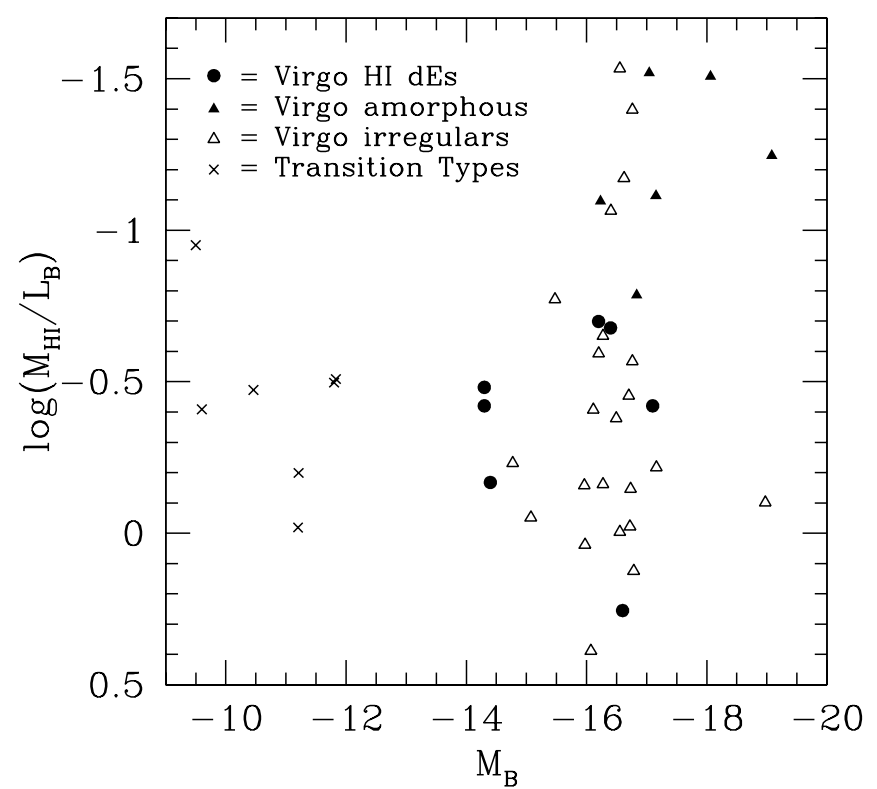

FIG. 11.-Absolute magnitude $\left(M_{B}\right)$ vs. $M_{\mathrm{H}_{\mathrm{I}}} / L_{B}$ diagram for various galaxy populations, including Virgo irregular and amorphous galaxies from Gallagher \& Hunter (1989), the dE H I sample studied in this paper, and dwarf transition types in the Local Group.

LMCGs are most similar to the Local Group transition types in terms of their $\mathrm{H}$ I gas and stellar properties.

We can also examine the differences between the Virgo dE galaxies with $\mathrm{H}$ I and Virgo Cluster dwarf irregular galaxies observed by Gallagher \& Hunter (1986, 1989). Figure 11 plots $M_{\mathrm{H}_{\mathrm{I}}} / L_{B}$ ratios versus $M_{B}$ for the $\mathrm{H}_{\mathrm{I}} \mathrm{LMCGs}$, nearby dwarf transition types, and the dwarf irregular and amorphous galaxies studied by Gallagher \& Hunter (1989), converted to our assumed distance of $18 \mathrm{Mpc}$. Figure 11 includes the Virgo amorphous galaxies, objects that morphologically appear to be early types but have evidence for star formation (Sandage \& Brucato 1979). Figure 11 shows that the Virgo LMCGs classified as dE galaxies with $\mathrm{H}$ I detections (filled circles) on average have $M_{\mathrm{H}} / L_{B}$ values between the values found for the gas-rich Virgo irregular galaxies and the amorphous galaxies, which are gasdepleted (see Table 5). The nearby dwarf transition types in the Local Group are plotted as crosses in Figure 11, and their $M_{\mathrm{H}_{\mathrm{I}}} / L_{B}$ distribution is very similar to the $M_{\mathrm{H}} / L_{B}$ ratios for $\mathrm{H}$ I LMCGs classified as dE galaxies.

\subsubsection{The Four LMCG Populations (Yet Known)}

We can gain further insight into LMCGs by examining the limited information we have on the stellar and gaseous properties of all the different types. Figure 12 shows the correlation between the $(U-B)$ colors and the $M_{\mathrm{H}_{\mathrm{I}}} / L_{B}$ ratios of the Virgo dwarf irregular and amorphous samples from Gallagher \& Hunter (1989). As Figure 12 shows, the more gas-depleted objects, as measured by the $M_{\mathrm{HI}} / L_{B}$ ratio, have redder $(U-B)$ colors, indicating the increasing dominance of older and possibly more metal-rich stellar populations. We also plot on Figure 12 the location of the two $\mathrm{H} \mathrm{I}$ LMCGs with $(B-V)$ color measurements, VCC 168 and VCC 608. We convert the $(B-V)$ colors for these objects into a $(U-B)$ color by fitting, and using, the relationship between these two colors for all galaxies in the Third Reference Catalogue of Bright Galaxies (de Vaucouleurs et al. 


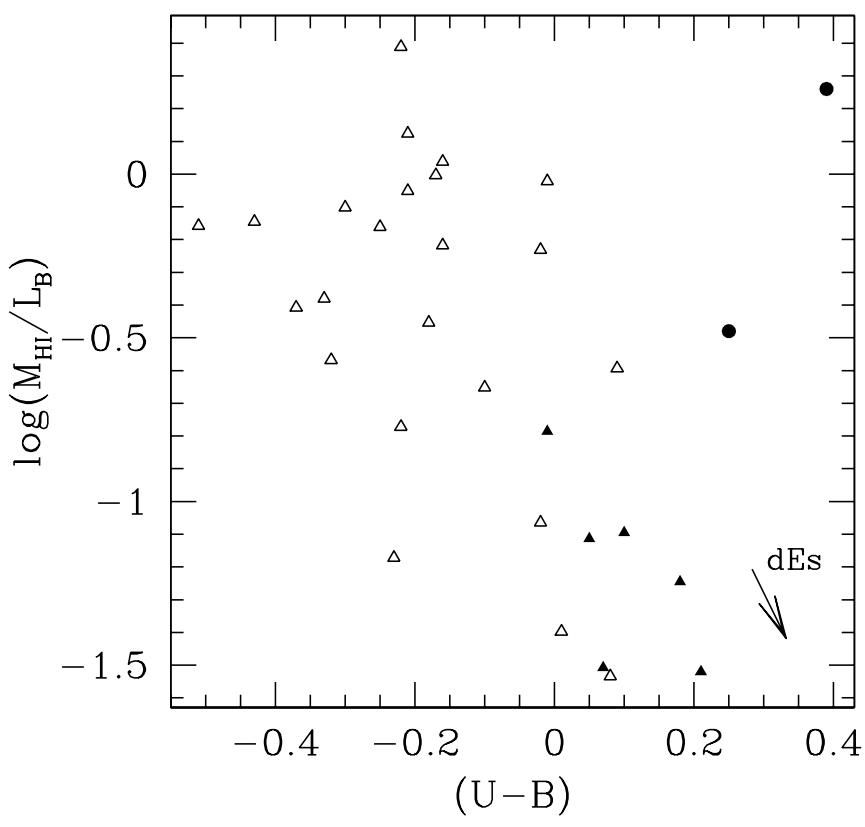

FIG. 12.-Diagram of $M_{\mathrm{H}} / L_{B}$ vs. $(U-B)$ color for the irregular (open triangles) and amorphous (filled triangles) Virgo galaxy sample from Gallagher \& Hunter (1986). The two H I-detected LMCGs with both $M_{\mathrm{H}} /$ $L_{B}$ and derived $(U-B)$ colors, VCC 168 and VCC 608, are plotted as filled circles.

1991): $(U-B)=1.26 \pm 0.01(B-V)-0.71 \pm 0.01$. The $\mathrm{H} \mathrm{I}-$ detected LMCGs have $M_{\mathrm{H}} / L_{B}$ values similar to those of Virgo dwarf irregular galaxies, but have red colors, similarly to the amorphous galaxies. It appears that these $\mathrm{H}$ I LMCGs might be members of a new class of LMCGs, pending proper $(U-B)$ color measurements. An arrow in Figure 12 shows where $\mathrm{dE}$ galaxies with no detectable $\mathrm{H}$ I would fall on this diagram, since they are red and gas-depleted systems.

Based on this, there appear to be at least four types of low-mass galaxies associated with the Virgo Cluster: (1) the classical dwarf irregular galaxies with active star formation and gas, which are likely in the process of being accreted into clusters (Gallagher \& Hunter 1989; Paper I), (2) dwarf elliptical galaxies with older stellar populations and $\mathrm{H}$ i gas masses less than $10^{6} M_{\odot}$ that were accreted more than $3 \mathrm{Gyr}$ ago, some as larger mass galaxies, or those that originally formed with the cluster (Paper III), (3) amorphous galaxies, and (4) the H I-rich early-type LMCGs studied in this paper. These last two types are likely LMCGs undergoing morphological evolution from accreted low-luminosity disks or irregular galaxies. The amorphous galaxies, because of their low $\mathrm{H}$ I content and red colors, are possibly systems that were accreted into eccentric orbits or are under physical conditions, such as within groups, where they lost a significant amount of their $\mathrm{H}$ I gas mass. H I LMCGs, discussed in this paper, are potentially $\mathrm{dE}$ galaxies that may have retained their $\mathrm{H}$ I gas because they were accreted into the cluster on high angular momentum orbits. The redder colors of VCC 168 and VCC 608 and the morphologies of the H I LMCGs both suggest that H I LMCGs have existed in the cluster for a longer time than the amorphous galaxies.

In the above picture we are not arguing that all dwarf elliptical galaxies originate from dwarf irregular galaxies, since the usual objections for this evolutionary process still hold (Paper I). The only dwarf elliptical galaxies/spheroids

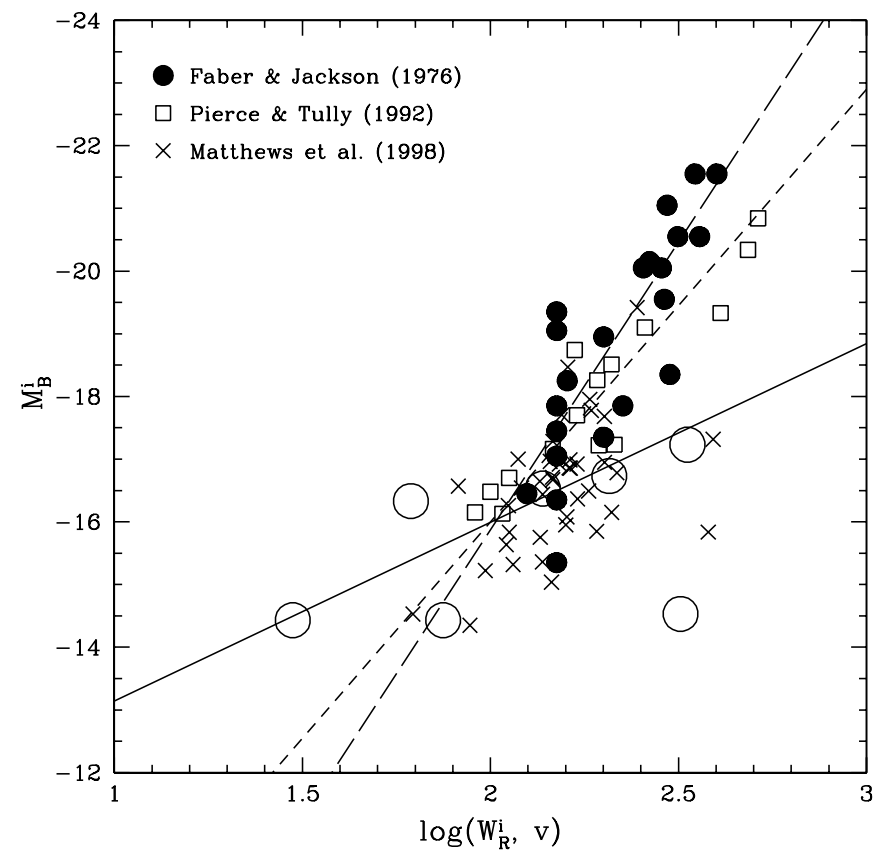

FIG. 13.-Velocity line width $\left(W_{R}^{i}\right)$ or velocity dispersion $(v)$ vs. luminosity $\left(M_{B}^{i}\right)$ relationship for various types of galaxies. The filled circles show the galaxies from the sample in Faber \& Jackson (1976), and the open sqaures show the sample used in Pierce \& Tully (1992). The long-dashed line shows a fit to the Faber-Jackson points, while the short-dashed line shows the Tully-Fisher relationship, and the crosses show the extreme latetype disk galaxies from Matthews et al. (1998). The open circles show the LMCGs after correcting their velocities for inclination and removing in quadrature a minimum value for turbulence, $W_{t}=5 \mathrm{~km} \mathrm{~s}^{-1}$.

that originate from the irregular galaxies would be those that are faint with low surface brightnesses. The origin of the brighter, higher surface brightness objects is potentially dynamical in nature (Papers I and III; Conselice 2002).

\subsection{The Luminosity-Line Width Relationship}

Figure 13 shows the luminosity-line width diagram for the H I LMCGs detections listed in Table 3, minus the two galaxies we conclude are either not likely a dE or a false $\mathrm{H} \mathrm{I}$ detection. With the exception of VCC 390, which has a high $M_{\text {dyn }} / L_{B}$ ratio of $\sim 45$, there is a general correlation between the absolute magnitude $M_{B}$ and the velocity width $W_{20}$. Also plotted in Figure 13 are the Faber-Jackson and TullyFisher (1977) relationships between $M_{B}$ and the velocity line width, or internal stellar velocity dispersion. Data from which these relationships are fitted are shown and are from Faber \& Jackson (1976) and Pierce \& Tully (1992). ${ }^{8}$ Also plotted in Figure 13, as crosses, are very late-type disk galaxies, which are good candidates for being LMCG progenitors, taken from Matthews, van Driel, \& Gallagher (1998).

The magnitude and velocity data for the $\mathrm{H}$ I LMCGs listed in Table 3 are plotted as open circles and represent the magnitudes and $W_{20}$ values after correcting for inclination and subtracting out a component to account for turbulence.

\footnotetext{
${ }^{8}$ We convert the magnitudes in the Faber \& Jackson (1976) photometry to match the Hubble constant, $85 \mathrm{~km} \mathrm{~s}^{-1} \mathrm{Mpc}^{-1}$, used in Pierce \& Tully (1992). The magnitudes for the spiral galaxies in Pierce \& Tully (1992) were corrected for internal and galactic extinction. The velocity widths, $W_{R}$, plotted for the spiral data are corrected using the methods outlined in Tully \& Fouqué (1985).
} 
While the turbulent velocity contribution could be fairly high in some galaxies (Tully \& Fouqué 1985; de Blok, McGaugh, \& van der Hulst 1996), it is likely lower in dwarf galaxies, $\sim 5 \mathrm{~km} \mathrm{~s}^{-1}$, based on measurements of nearby low-mass galaxies (see, e.g., Young \& Lo 1997). We therefore remove $5 \mathrm{~km} \mathrm{~s}^{-1}$ from the $W_{20}$ velocity widths when correcting for turbulence.

The solid line shows a best-fit relationship between the galactic extinction-corrected $M_{B}$ values and velocity widths for the H I LMCGs. This fit shows that the LMCGs classified as $\mathrm{dE}$ galaxies with $\mathrm{H}$ I gas appear to deviate from the Tully-Fisher and Faber-Jackson relationships in both slope and zero point and are more similar to the trends, shallower slope, and individual values found for the extreme late types in the Matthews et al. (1998) sample. This relationship is also fairly flat, and several galaxies clearly deviate from it. Figure 13 shows that the galaxy sample under consideration, while displaying a luminosity-line width correlation on average (solid line), has a fundamentally different nature than the relationship for both the spiral and elliptical galaxies. While, e.g., Matthews et al. (1998) find that some faint galaxies have high velocities for their magnitudes compared to Tully-Fisher, revealing a possible high mass-to-light ratio, we do not generally see this trend for all the $\mathrm{H} \mathrm{I-}$ detected LMCGs.

There are several possible ways to account for the dispersion in the relationship between $\mathrm{H}$ I line width and absolute magnitude in the $\mathrm{H}$ I-detected early-type LMCGs besides observational error. One is that these galaxies are in a mix of evolutionary stages, such that the velocity width is sensitive to not only the mass of the galaxy but also whether or not the gas disk has been extended or truncated by cluster processes, such as gas and tidal stripping (Moore et al. 1998). Models of the evolution of low-mass galaxies in groups also show that as a galaxy interacts with a larger system and loses mass, its mass-to-light ratio varies with time depending on whether any new star formation occurs and what the mass profiles of the various components are (see, e.g., Mayer et al. 2001). The mass-to-light ratio could also be somewhat overestimated by material stripped from galaxies that can produce a larger observed velocity dispersion and a range of derived mass-to-light ratios (Mayer et al. 2001). This would, however, require nonvirial motions of the gas, which are unlikely if ram pressure forces are reduced in intensity $(\S 4.3)$.

\section{THE RECENT VIRGO ACCRETION RATE}

We can use the information on the number of transitiontype dwarf galaxies in the Virgo Cluster to get an idea of the recent galaxy accretion rate that might be responsible for producing these objects. To obtain this number, we follow the procedure presented by us in Gallagher, Conselice, \& Wyse (2001).

Using Gallagher et al. (2001) and assuming a cluster age of $10 \mathrm{Gyr}$ and a dE production efficiency from all but giant galaxies of $100 \%$, and since there are $\sim 10^{3} \mathrm{dE}$ galaxies in Virgo with $M_{B} \leq-14$, we derive an average infall rate of $\geq 100$ galaxies $\mathrm{Gyr}^{-1}$. High infall rates in the past are therefore necessary to populate modern clusters with low-mass cluster objects, but higher past cluster infall rates are likely in a low- $\Omega_{M}$ universe (Kauffmann 1995).

Infall rates are difficult to measure and probably will be episodic, since galaxies are observed to accrete into Virgo in groups (see, e.g., Tully \& Shaya 1984). One estimate can be derived from the Gallagher \& Hunter (1989) sample that suggests that a few dozen galaxies are currently undergoing morphological transformations. This is similar to the $\sim 20$ galaxies estimated to have recently fallen into Virgo in the last Gyr (Tully \& Shaya 1984). We obtain another estimate of the total number of current transition dwarf objects in the Virgo Cluster using the results of this paper. A total of 48 objects classified as dwarf elliptical galaxies were observed for $\mathrm{H}$ I, of which a total of seven are likely transition objects. If we assume that these 48 are representative of the entire Virgo dwarf elliptical population and ignore any transition types that may be classified as dwarf irregular galaxies, then $\sim 15 \%$ of all dwarf elliptical galaxies with $M_{B}<-14$ in the Virgo Cluster are in some kind of transition phase. Since there are roughly $10^{3}$ dwarf elliptical galaxies in the Virgo Cluster (VCC), then we expect $\sim 150$ of these galaxies to be in transition throughout Virgo. If we assume that an average transition phase takes 1-3 Gyr (see, e.g., Moore et al. 1998), then the recent galaxy infall rate in the last $3 \mathrm{Gyr}$ (from $z \sim 0.2$ in $\Lambda \mathrm{CDM}$ ) is $\dot{N}_{c}\left(t_{0}\right) \approx 150$ galaxies $3 \mathrm{Gyr}^{-1} \sim 50$ galaxies $\mathrm{Gyr}^{-1}$. This is an upper bound since some mixed-structure galaxies could be on nearly circular orbits and thus slowly evolving.

We can use further assumptions about the average amount of mass per galaxy to derive a mass infall rate of dwarf galaxies into the Virgo Cluster in the last 3 Gyr. The average absolute magnitude for the $\mathrm{H} \mathrm{I}$-detected LMCGs is $M_{B}=-15.6 \pm 1.2$. This gives an average mass per galaxy, assuming a stellar mass-to-light ratio of 5 in solar units, of $\sim 5 \times 10^{8} M_{\odot}$. Using this approximation for our hypothetical 150 transition dwarf galaxies accreted over the last $3 \mathrm{Gyr}$ gives a mass influx into the Virgo Cluster of $\sim 50 M_{\odot} \mathrm{yr}^{-1}$ only from this low-mass component. Assuming a normal luminosity function for infalling galaxies, the total timeaveraged infall rate would be several times larger than this, $\sim 200-300 M_{\odot} \mathrm{yr}^{-1}$. While we have used a very simplistic model to obtain this result, it shows that an accretion model for H I LMCGs evolving into dwarf elliptical galaxies gives reasonable galaxy and mass infall rates, comparable to other Virgo Cluster accretion estimates (see, e.g., Tully \& Shaya 1984).

Are these calculations of the recent and past accretion history into clusters consistent with cosmological model predictions? In a $\Lambda$-dominated flat cosmology, the linear growth of structure slows down at intermediate redshift, $1+z \sim \Omega_{m}^{-1 / 3}$ (note that this is a lower redshift than that at which linear growth slows for a matter-dominated open universe with the same $\Omega_{m}$ ), but growth is a process that continues until the present day, with larger structures assembling a larger fraction of their mass later. Simulations designed to constrain cosmological parameters through the analysis of the amplitude of substructure in clusters (Crone et al. 1996) have shown that in a model with $\Omega_{\Lambda}=0.8, \Omega_{m}=0.2$, and a power spectrum similar to that of CDM over cluster mass scales $(n=-1), 50 \%$ of the mass of a typical large cluster was assembled within the last $5 h^{-1}$ Gyr. The $\Lambda$ CDM simulations of Wechsler et al. (2002) also show continuous accretion and mass assembly to the present day, with their most massive halos, $M>3 \times 10^{13} h^{-1} M_{\odot}$, having somewhat lower acccretion than a typical galaxy cluster, accreting around $25 \%$ of their mass since $z \sim 0.7$ (their Fig. $4 a$ ), or a look-back time of $\sim 7$ Gyr. These halos accrete typically about $10 \%$ of their mass in the last $\sim 3 \operatorname{Gyr}(z \sim 0.25)$. The 
"Virgo-like" clusters simulated by Governato, Ghigna, and Moore (2001) also accrete $\sim 20 \%$ of their mass since $z=0.25$ in the "concordance" $\Lambda \mathrm{CDM}$ universe (see their Fig. 5; a similar recent accretion history is found in the open CDM model). These CDM models are therefore consistent with the idea that Virgo H I-rich early-type LMCGs are a recently accreted galaxy population.

\section{SUMMARY AND CONCLUSIONS}

In this paper we present evidence that $\mathrm{H}$ I-rich Virgo Cluster dE galaxies, discovered through an Arecibo $21 \mathrm{~cm}$ and WIYN $3.5 \mathrm{~m}$ telescope study, form a new class of low-mass galaxies.

1. We find two new low-mass galaxy $\mathrm{H}$ i $21 \mathrm{~cm}$ line detections with the Arecibo telescope in the Virgo Cluster, out of 22 observed objects classified as dE galaxies with optically determined radial velocities. These dwarf galaxies, VCC 390 and VCC 1713, have $M_{\mathrm{HI}}=6 \times 10^{7}$ and $8 \times 10^{7} M_{\odot}$, respectively. The other 20 Virgo dE members we observed were not detected, with $3 \sigma$ upper limits of $M_{\mathrm{H}_{\mathrm{I}}} \leq 8 \times 10^{6}$ $M_{\odot}$, corresponding to $M_{\mathrm{H}} / L_{B}<0.1$ (see Table 1).

2. A survey of the literature yields an additional five Virgo early-type dwarf galaxies with credible H I measurements, for a total of seven detections among 48 galaxies with sensitive $21 \mathrm{~cm}$ line observations (Table 2). About half of all Virgo dE galaxies with measured radial velocities (see Paper I) now have been searched for $\mathrm{H}$ I to the $\sim 10^{7} M_{\odot}$ level with a $15 \% \mathrm{H}$ I detection rate.

3. Optical imaging with the WIYN telescope for five of these Virgo early-type LMCGs with $21 \mathrm{~cm}$ detections allowed us to perform morphological classifications using quantitative techniques. One of our new detections, VCC 1713 , shows clumpy substructure, which could be due to internal dust or residual effects of star formation; it appears to be in a relatively early stage of transition from a starforming galaxy to a dE-like system. We further suspect that VCC 2062 is a dIrr galaxy, since it has a faint, patchy structure on DSS images, and we removed it from the early-type Virgo LMCG sample. The morphologies and other known optical structural characteristics of the remaining five $\mathrm{H} \mathrm{I}-$ detected galaxies are consistent with those of normal $\mathrm{dE}$ members of the Virgo Cluster. The optical spectrum of one of our detections, VCC 390 (Fig. 4), is also very similar to other known dE spectra. The Virgo H I-rich LMCGs have gas masses well above the levels found in Local Group $\mathrm{dE}$ galaxies, e.g., NGC 185 and NGC 205 . These relatively gasrich galaxies may constitute $\sim 15 \%$ of Virgo's moderateluminosity population of early-type dwarf galaxies.

4. The early-type LMCGs in Virgo with $\mathrm{H}$ I are found preferentially near the edge of the cluster, at radii $\geq 0.5 \mathrm{Mpc}$ from the cluster center, and have a flat distribution of observed radial velocities, suggesting that they have recently been accreted (and not crossed the cluster yet) or are on high angular momentum orbits and are possibly accreted in groups.

5. We show that the H I LMCGs studied in this paper have $\mathrm{H}$ I properties more similar to those of Local Group transition-type dwarf galaxies or dwarf irregular galaxies rather than $\mathrm{dE}$ galaxies or Virgo amorphous galaxies.

We use this observational information, along with models of various cluster-driven galaxy evolutionary processes, especially gas removal due to ram pressure stripping and Kelvin-Helmholtz instabilities, to conclude the following about these H I LMCGs and other low-mass cluster galaxies in Virgo:

1. Based on the positions of H I LMCGs in the outskirts of Virgo and their high velocities relative to the cluster, we conclude that some, or all, of the H I-rich Virgo dE galaxies are on high angular momentum orbits and therefore never go through the cluster core. This is a natural explanation for how these galaxies are able to keep their $\mathrm{H}$ I gas over long time spans. If these galaxies had highly eccentric orbits and passed through the Virgo center, they would be rapidly stripped of their gas in less than a cluster crossing time. Calculations of gas loss due to ram pressure and KelvinHelmholtz instabilities show that these galaxies would be depleted of $\mathrm{H}$ I gas, even at their projected position, over $\sim 10$ Gyr but can potentially keep their gas when on high angular momentum orbits for up to a few Gyr.

2. Through a comparison of gas properties of Local Group dwarf elliptical galaxies and spheroidal galaxies, which have much lower gas content than the Virgo H I-rich LMCGs classified as dE galaxies, we conclude that these galaxies must have been of a different morphological type in the past. This is consistent with the idea that these galaxies were accreted as gas-rich star-forming systems into Virgo during the last $1-3 \mathrm{Gyr}$, since this is approximately the same amount of time needed to convert a disk or irregular galaxy with star formation into a red early-type dwarf. Even at the present epoch, galaxy clusters act as engines of evolutionary change. They effectively strip gas and stars from later-type low-mass galaxies captured from the cluster surroundings and thereby could convert low-luminosity disk galaxies and irregular galaxies into early-type cluster dwarf galaxies. We also find two very high $\mathrm{H}$ I line widths that need to be explained.

3. Combining this analysis with the dwarf irregular and amorphous galaxy properties from Gallagher \& Hunter (1989) and Paper I, we can sketch a possible evolutionary/ formation scenario for LMCGs in Virgo. The dwarf irregular galaxies are recent additions into clusters that have not yet crossed through the cluster, but when they do, they will be stripped of much of their gas. The presence of $\mathrm{H} \mathrm{I}$ in some Virgo LMCGs classified as dE galaxies is additional evidence that some LMCGs are created from infalling field galaxies (Papers I and III). Other dwarf elliptical galaxies in Virgo have likely been present since the cluster was formed. We argue that the amorphous and H I LMCGs are both accreted populations, either former disk galaxies or dwarf irregular galaxies, whose evolution has diverged because of their orbits in the cluster. The amorphous galaxies are possibly on radial orbits, or systems in previous collisions (Gallagher \& Hunter 1989), whose H I gas has been stripped and are now passively evolving into redder systems. The $\mathrm{H}$ I LMCGs are, in our scenario, systems on circular orbits whose $\mathrm{H}$ I gas remains and whose stellar populations and morphologies evolved to resemble dwarf elliptical galaxies. In this model, the $\mathrm{H}$ I LMCGs were accreted before the amorphous galaxies to allow time for morphological evolution.

4. A simple calculation of expected Virgo Cluster infall rates based on the number of low-mass galaxies observed in evolutionary transition phases yields reasonable results, with a derived average recent mass infall rate of $\dot{M} \sim 50 M_{\odot}$ 
$\mathrm{yr}^{-1}$ and most likely a time-averaged rate of $200-300 M_{\odot}$ $\mathrm{yr}^{-1}$ when giant galaxies are included.

We thank Chris Salter for his invaluable assistance in obtaining the data presented in this paper and for his generous hospitality while C. J. C. was observing at Arecibo. We also thank Lynn Matthews for help in taking the WIYN images and for supplying us with velocities and magnitudes of her low-mass disk galaxy sample. The anonymous referee made many valuable points after a careful and thorough reading of this paper. This research was supported in part by the National Science Foundation (NSF) through grants AST 98-03018 to the University of Wisconsin at Madison and AST 98-04706 to Johns Hopkins University. C. J. C. acknowledges support from an NSF Astronomy and Astrophysics Postdoctoral Fellowship, a Grant-In-Aid of Research from Sigma Xi and the National Academy of Sciences (NAS), as well as a Graduate Student Researchers Program (GSRP) Fellowship from NASA and the Graduate Student Program at the Space Telescope Science Institute.
Abadi, M. G., Moore, B., \& Bower, R. G. 1999, MNRAS, 308, 947 Aguilar, L. A., \& White, S. D. M. 1985, ApJ, 295, 374 Almoznino, E., \& Brosch, N. 1998, MNRAS, 298, 920

Baars, J. W. M., Genzel, R., Pauliny-Toth, I. I. K., \& Witzel, A. 1977, A\&A, 61, 99

Bershady, M. A., Jangren, A., \& Conselice, C. J. 2000, AJ, 119, 2645

Binggeli, B., \& Cameron, L. M. 1993, A\&AS, 98, 297

Binggeli, B., Tammann, G. A. \& Sandage, A. 1987, AJ, 94, 251 (VCC)

Blumenthal, G. R., Faber, S. M., Primack, J. R., \& Rees, M. J. 1984, Nature, 311,517

Bothun, G. D., Mould, J. R., Wirth, A., \& Caldwell, N. 1985, AJ, 90, 697

Bottinelli, K., Gouguenheim, L., Fouque, P., \& Paturel, G. 1990, A\&AS, 82,391

Broeils, A. H. 1992, Ph.D. thesis, Univ. Groningen

Burstein, D., Krumm, N., \& Salpeter, E. E. 1987, AJ, 94, 883

Cayatte, V., van Gorkum, J. H., Balkowski, C., \& Kotanyi, C. 1990, AJ, 100,604

Cavaliere, A., \& Fusco-Fermiano, R. 1976, A\&A, 49, 137

Conselice, C. J. 1997, PASP, 109, 1251 2002, ApJ, 573, 5L 2003, ApJS, 147,

Conselice, C. J., Bershady, M. A., \& Gallagher, J. S. 2000a, A\&A, 354, 21 L

Conselice, C. J., Bershady, M. A., \& Jangren, A. 2000b, ApJ, 529, 886

Conselice, C. J. \& Gallagher, J. S. 1998, MNRAS, 297, 34L 1999, AJ, 117, 75

Conselice, C. J., Gallagher, J. S., \& Wyse, R. F. G. 2001a, ApJ, 559, 791 (Paper I) $2001 \mathrm{~b}, \mathrm{AJ}, 122,228$ 2002, AJ, 123, 2246 (Paper II) 2003, AJ, 125, 66 (Paper III)

Couch, W. J., Barger, A. J., Smail, I., Ellis, R. S., \& Sharples, R. M. 1998, ApJ, 497, 188

Crone, M. M., Evrard, A. E., \& Richstone, D. O. 1994, ApJ, 434, 402

de Blok, E., McGaugh, S. S., \& van der Hulst, J. M. 1996, MNRAS, 283, 18 de Vaucouleurs, G., de Vaucouleurs, A Corwin, H. G., Buta, R. J.,

Paturel, G., \& Fouque, P. 1991, Third Reference Catalaogue of Bright

Galaxies (Berlin: Springer)

Dressler, A. 1986, ApJ, 301, 35

Dressler, A., et al. 1997, ApJ, 490, 577

Duprie, K., \& Schneider, S. E. 1996, AJ, 112, 937

Faber, S. M., \& Gallagher, J. S. 1976, ApJ, 204, 365

Faber, S. M., \& Jackson, R. E. 1976, ApJ, 204, 668

Ferguson, H. C., \& Binggeli, B. 1994, A\&A Rev., 6, 67

Gallagher, J. S., Conselice, C. J., \& Wyse, R. F. G. 2001, in Dwarf Galaxies and Their Environment, ed. K. S. de Boer, R. J. Dettmar, \& U. Klein (Aachen: Shaker), 213

Gallagher, J. S. \& Hunter, D. A. 1984, ARA\&A, 22, 37 1986, AJ, 92, 557 1989, AJ, 98, 806

Gallagher, J. S., \& Wyse, R. F. G. 1994, PASP, 106, 1225

Gavazzi, G., Zibetti, S., Boselli, A., Franzetti, P., Scodeggio, M., \& Martocchi, S. 2001, A\&A, 372, 29

Ghigna, S., Moore, B., Governato, F., Lake, G., Quinn, T., \& Stadel, J. 1998, MNRAS, 300, 146

Gnedin, O. Y., Zhao, H., Pringle, J. E., Fall, S. M., Livio, M., \& Meylan, G. 2002, ApJ, 568, 23L

Governato, F., Ghigna, S., \& Moore, B. 2001, in ASP Conf. Ser. 245, Astrophysical Ages and Timescales, ed. T. von Hippel, C. Simpson, \& N. Manset (San Francisco: ASP), 469

Grogin, N. A., Geller, M. J., \& Huchra, J. P. 1998, ApJS, 119, 277

Gunn, J. E., \& Gott, J. R. 1972, ApJ, 176, 1

Harris, J., Calzetti, D., Gallagher, J. S., Conselice, C. J., \& Smith, D. A. 2001, AJ, 122, 3046

Haynes, M. P., \& Giovanelli, R. 1986, ApJ, 306, 466

Heller, A., Almoznino, E., \& Brosch, N. 1999, MNRAS, 304, 8

\section{EFERENCES}

Hibbard, J. E. \& Sansom, A. E. 2003, AJ, 125, 667

Hoffman, G. L., Helou, G., Salpeter, E. E., Glosson, J., \& Sandage, A. 1987, ApJS, 63, 247

Hoffman, G. L., Lu, N. Y., Salpeter, E. E., Farhat, B., Lamphier, C., \& Roos, T. 1993, AJ, 106, 39

Huchtmeier, W. K. 1979, A\&A, 75, 170

Huchtmeier, W. K., \& Richter, O.-G. 1986, A\&AS, 64, 111

Huss, A., Jain, B. \& Steinmetz, M. 1999, MNRAS, 308, 1011

Johnson, D. W., \& Gottesman, S. T. 1983, ApJ, 275, 549

Kauffmann, G. 1995, MNRAS, 274, 153

Knapp, G. R., Kerr, F. J., \& Bowers, P. F. 1978, AJ, 83, 360

Knezek, P. M. Sembach, K. R. \& Gallagher, J. S. III. 1999, ApJ, 514, 119

Koribalski, B., Johnston, S., \& Ortupcek, R. 1994, MNRAS, 270, 43L

Kuehr, H., Witzel, A., Pauliny-Toth, I. I. K., Nauber, U. 1981, A\&AS, 45, 367

Lake, G., \& Schommer, R. A. 1984, ApJ, 280, 107

Lewis, B. M., Helou, G., \& Salpeter, E. E. 1985, ApJS, 59, 161

Mao, S., \& Mo, H. J. 1998, MNRAS, 296, 847

Mateo, M. L. 1998, ARA\&A, 36, 435

Matthews, L., van Driel, W. \& Gallagher, J. S. 1998, AJ, 116, 2196

Mayer, L., Governato, F., Colpi, M., Moore, B., Quinn, T., Wadsley, J., Stadel, J., \& Lake, G. 2001, ApJ, 559, 754

Moore, G., Lake, G., \& Katz, N. 1998, ApJ, 495, 139

Mori, M. \& Burkert, A. 2000, ApJ, 538, 559

Nulsen, P. E. J. 1982, 1982, MNRAS, 198, 1007

Oemler, A., Dressler, A., \& Butcher, H. R. 1997, ApJ, 474, 561

Pierce, M. J., \& Tully, R. B. 1992, ApJ, 387, 47

Quilis, V., Moore, B., \& Bower, R. 2000, Science, 288, 1617

Roberts, M. S., \& Haynes, M. P. 1994, ARA\&A, 32, 115

Ryden, B., Terndrup, D. M., Pogge, R. W., \& Lauer, T. R. 1999, ApJ, 517, 650

Sadler, E. M., Oosterloo, T. A., Morganti, R., \& Karakas, A. 2000, AJ, 119,1180

Sage, L. J., Welch, G. A., \& Mitchell, G. F. 1998, ApJ, 507, 726

Sandage, A., Binggeli, B., \& Tammann, G. A. 1985, AJ, 90, 1759

Sandage, A., \& Brucato, R. 1979, AJ, 84, 472

Schneider, S. E., Thuan, T. X., Magri, C., \& Wadiak, J. E. 1990, ApJS, 72, 245

Solanes, J. M., Marique, A., García-Gómez, C., González-Casado, G., Giovanelli, R., \& Haynes, M. 2001, ApJ, 548, 97

Spitzer, L. 1958, ApJ, 127, 17

Trentham, N., Tully, R. B., \& Verheijen, M. A. W. 2001, MNRAS, 325, 385

Tully, R. B., \& Fisher, J. R. 1977, A\&A, 54, 661

Tully, R. B., \& Fouqué, P. 1985, ApJS, 58, 67

Tully, R. B., \& Shaya, E. J. 1984, ApJ, 281, 31

van den Bergh, S. 2000, The Galaxies of the Local Group (Cambridge: Cambridge Univ. Press)

van Driel, W., Ragaigne, D., Boselli, A., Donas, J., \& Gavazzi, G. 2000 A\&AS, 144,463

van Driel, W. \& van Woerden, H. 1989, A\&A, 225, 317

van Zee, L., Haynes, M. P., \& Giovanelli, R. 1995, AJ, 109, 990

van Zee, L., Skillman, E. D., \& Salzer, J. J. 1998, AJ, 116, 1186

Vigroux, L., Lachieze-Rey, M., Thuan, T. X., \& Vader, J. P. 1986, AJ, 91, 70

Volders, L. M. J. S., \& Högbom, J. A. 1961, Bull. Astron. Inst. Netherlands, 15, 307

Vollmer, B., Cayatte, V., Balkowski, C., \& Duschl, W. J. 2001, ApJ, 561, 708

Wechsler, R. H., Bullock, J., Primack, J. R., Kravtsov, A. V., \& Dekel, A. 2002, ApJ, 568, 52

White, S. D. M., \& Frenk, C. S. 1991, ApJ, 379, 52

Worthey, G. 1994, ApJS, 95, 107

Young, L. M. 1999, AJ, 117, 1758

Young, L. M., \& Lo, K. Y. 1997, ApJ, 476, 127 